\title{
NEW RESULTS IN THE PERTURBATION THEORY OF MAXIMAL MONOTONE AND $M$-ACCRETIVE OPERATORS IN BANACH SPACES
}

\author{
ATHANASSIOS G. KARTSATOS
}

\begin{abstract}
Let $X$ be a real Banach space and $G$ a bounded, open and convex subset of $X$. The solvability of the fixed point problem (*) Tx+Cx $\ni x$ in $D(T) \cap \bar{G}$ is considered, where $T: X \supset D(T) \rightarrow 2^{X}$ is a possibly discontinuous $m$-dissipative operator and $C: \bar{G} \rightarrow X$ is completely continuous. It is assumed that $X$ is uniformly convex, $D(T) \cap G \neq \emptyset$ and $(T+C)(D(T) \cap \partial G) \subset \bar{G}$. A result of Browder, concerning single-valued operators $T$ that are either uniformly continuous or continuous with $X^{*}$ uniformly convex, is extended to the present case. Browder's method cannot be applied in this setting, even in the single-valued case, because there is no class of permissible homeomorphisms. Let $\Gamma=\left\{\beta: \mathcal{R}_{+} \rightarrow \mathcal{R}_{+} ; \beta(r) \rightarrow 0\right.$ as $\left.r \rightarrow \infty\right\}$. The effect of a weak boundary condition of the type $\langle u+C x, x\rangle \geq-\beta(\|x\|)\|x\|^{2}$ on the range of operators $T+C$ is studied for $m$-accretive and maximal monotone operators $T$. Here, $\beta \in \Gamma, x \in D(T)$ with sufficiently large norm and $u \in T x$. Various new eigenvalue results are given involving the solvability of $T x+\lambda C x \ni 0$ with respect to $(\lambda, x) \in(0, \infty) \times D(T)$. Several results do not require the continuity of the operator $C$. Four open problems are also given, the solution of which would improve upon certain results of the paper.
\end{abstract}

\section{Introduction. Preliminaries}

The symbol $X$ stands for a real Banach space with norm $\|\cdot\|$ and (normalized) duality mapping $J$. In what follows, "continuous" means "strongly continuous". We denote by $\mathcal{R}, \mathcal{R}_{+}$the sets $(-\infty, \infty),[0, \infty)$, respectively. The symbols $\operatorname{int} D, \partial D$ and $\bar{D}$ denote the strong interior, boundary and closure of the set $D$, respectively. An operator $T: X \supset D(T) \rightarrow Y$, with $Y$ another real Banach space, is "bounded" if it maps bounded subsets of $D(T)$ onto bounded sets. It is "compact" if it is continuous and maps bounded subsets of $D(T)$ onto relatively compact sets. It is "demicontinuous" if it maps strongly convergent sequences into weakly convergent sequences. It is "completely continuous" if it maps weakly convergent sequences into strongly convergent sequences. For an operator $T: X \rightarrow 2^{Y}$ we set $D(T) \equiv\{x \in$ $X: T x \neq \emptyset\}$. We always assume that $D(T) \neq \emptyset$, for every multi-valued operator $T$ considered in this paper. Given $T: X \supset D(T) \rightarrow 2^{Y}$ and a set $G \subset D(T)$, we

Received by the editors February 7, 1995.

1991 Mathematics Subject Classification. Primary 47H17; Secondary 47B44, 47H09, 47H10.

Key words and phrases. $m$-accretive operator, maximal monotone operator, compact perturbation, compact resolvent, eigenvalues for nonlinear operators, fixed point theory, degree theory.

The results of this paper were announced in a lecture at the International Conference on Nonlinear Differential Equations, Kiev, Ukraine, August 21-27, 1995. 
set $T G=\bigcup\{T x: x \in G\}$. An operator $T: X \supset D(T) \rightarrow 2^{X}$ is "accretive" if for every $x, y \in D(T)$ there exists $j \in J(x-y)$ such that

$$
\langle u-v, j\rangle \geq 0 \text { for every } u \in T x, v \in T y .
$$

An accretive operator $T$ is "strongly accretive" if 0 in the right-hand side of (1) is replaced by $\alpha\|x-y\|^{2}$, where $\alpha>0$ is a fixed constant. In an obvious way, we define the concept of strong accretiveness on any subset of $D(T)$. An accretive operator $T$ is called " $m$-accretive" if $R(T+\lambda I)=X$ for every $\lambda>0$, where $I$ denotes the identity operator on $X$. It is called " $m$-dissipative" if $-T$ is $m$ accretive. We denote by $B_{r}(0)$ the open ball of $X$ (or $X^{*}$ ) with center at zero and radius $r>0$. For an $m$-accretive operator $T$, the "resolvents" $J_{\lambda}: X \rightarrow D(T)$ of $T$ are defined by $J_{\lambda}=(I+\lambda T)^{-1}$ for all $\lambda \in(0, \infty)$. $J_{\lambda}$ is a non-expansive mapping on $X$ for all $\lambda>0$. Also the operator $T_{\lambda} \equiv(1 / \lambda)\left(I-J_{\lambda}\right)$ is a globally Lipschitzian mapping with $T_{\lambda} x \in T J_{\lambda} x$, for every $x \in X$. For facts involving accretive operators, and other related concepts, the reader is referred to Barbu [2], Browder [5], Cioranescu [7], Deimling [9], and Lakshmikantham and Leela [31]. For a survey paper on compactness and accretivity, we cite the paper [23].

For a bounded set $\Omega \subset X$, the Kuratowski measure of noncompactness, $\gamma(\Omega)$, is defined by $\gamma(\Omega)=\inf \{\epsilon>0: \Omega$ can be covered by a finite family of sets of diameter $<\epsilon\}$.

A continuous mapping $g: X \supset D(g) \rightarrow X$ is called "condensing" if, for every non-empty, bounded and non-compact subset $\Omega$ of $D(g), g(\Omega)$ is bounded and $\gamma(g(\Omega))<\gamma(\Omega)$. Browder gave in [5, Theorem 13.23] the following result.

Theorem A. Let $X$ be a real Banach space and $G$ a bounded, convex, open subset of $X$. Let $T: \bar{G} \rightarrow X$ and $C: \bar{G} \rightarrow X$ be two mappings with $T$ accretive and $C$ compact. Suppose that either $T$ is locally uniformly continuous or $T$ is continuous and $X^{*}$ is uniformly convex. Then if $[I-(T+C)] \partial G \subset \bar{G}$ and $(T+C)(\bar{G})$ is closed, there exists $x \in \bar{G}$ such that $(T+C) x=0$.

One of our intentions in this paper is to give an analogous result, Theorem 1, for possibly multi-valued and discontinuous $m$-accretive operators $T: X \supset D(T) \rightarrow$ $2^{X}$. We then use this result to obtain a new fixed point theorem, Corollary 1 , for a mapping of the type $T+C$, where $T$ is now $m$-dissipative. It should be mentioned that Browder's method cannot be applied in our result (even in the single-valued case) because a discontinuous, strongly accretive operator does not belong to a class of permissible homeomorphisms as in Proposition (13.14) of [5]. On the other hand, one could take $T$ to be just a continuous accretive mapping in Theorem A. This is possible because Deimling's result in [8] says that a continuous strongly accretive mapping maps open sets of its domain onto open sets. It thus belongs to a class of permissible homeomorphisms.

In the proof of Theorem 1 in Section 2, we discovered that we had the ingredients for a new surjectivity result involving a boundary condition of the type

$$
\langle u+C x, j\rangle \geq-\beta(\|x\|)\|x\|^{2},
$$

for all $x \in D(T)$ with sufficiently large norm, all $u \in T x$ and some $j \in J x$. The function $\beta(\rho)$ here tends to zero as $\rho \rightarrow \infty$. This boundary condition can be combined with a growth condition on the sum $T+C$ in order to produce results of the type $\overline{R(T+C)}=X$ and $R(T+C)=X$. 
In Section 2 we also examine the existence of certain eigenvalues for the pair $(T, C)$. These eigenvalues equal $\lambda+1$, where $\lambda$ appears in the boundary conditions involving $T, C$ and in the assumption of compactness of the operator $C(\lambda T+I)^{-1}$. Theorem 4 utilizes this eigenvalue situation in order to produce a new result involving the existence of zeros for the sum $T+C$.

Section 3 contains the extension of some results of Section 2 to the case of maximal monotone operators $T: X \supset D(T) \rightarrow 2^{X^{*}}$. Theorem 6 shows how to extend Theorem 2 above to this setting. Theorem 7 involves the extendability of the main result of the author in [28]. That result was given for $m$-accretive operators. Our method here involves the use of the Brézis-Crandall-Pazy approximants $J_{\lambda}, T_{\lambda}$ introduced in [4]. There are some further differences between the proof of the result in [28] and that of Theorem 7. Theorem 9 solves a problem according to which an entire set lies in the range of the sum $T+C$. This interesting result extends a result in [29] and leads to Corollary 6 , which is a new surjectivity result for completely continuous perturbations of maximal monotone operators.

Section 4 is devoted to the problem of the existence of positive eigenvalues for the pair $(T, C)$. Theorem 9 there improves the maximal monotone analogue of Theorem 2.5 in [16], while Theorem 11 improves Theorem 3.3 in [16]. Both results make use of the above approximants from [4] and further demonstrate the usefulness of such a methodology.

In Section 5, we have decided to introduce the mapping $\widetilde{J} x \equiv\|x\|^{p} x$ in the study of nonlinear problems in real Hilbert spaces $H$. This mapping, whose finitedimensional versions have been used by a host of authors, was introduced in [30] in order to study certain perturbations of control problems involving accretive operators. In order to demonstrate the effectiveness of this method, we give a result which extends Theorem 2 (Section 2) and Theorem 6 (Section 3). This result, Theorem 12 , yields a criterion for the surjectivity of the sum $T+C$ which involves the number $p$ itself. We thus actually have a one-parameter family of surjectivity criteria in Hilbert spaces.

Section 6 contains some discussion on the results of this paper as well as five examples of operators $T$ or $C$ which can be easily used in order to apply several results herein to partial differential equations.

Finally, we have given four open problems, the solution of which would lead to the improvement of related results in this paper.

The results of this paper, as well as a good number of papers in the references, have applications in the control theory with preassigned responses (cf. Kaplan and the author [20] and the author and Mabry [30]) and the construction of methods of lines (cf. [24]). For other recent results of this nature, we refer to the papers by Ding and the author [10-11], Guan [13-14], Guan and the author [15-17], Hirano and Kalinde [19] and the author [22-24].

\section{Perturbations of $m$-Accretive Operators}

The following lemma is an extension of a lemma found in [15]. That lemma is more general than a lemma used by the author in [22] and [24]. We need a more localized version of it. In [15], it was assumed that $C: \overline{D(T)} \rightarrow X$.

Lemma 1. Let $X$ be uniformly convex. Let $T: X \supset D(T) \rightarrow 2^{X}$ be m-accretive 
and $C: \overline{D(T)} \supset \bar{G} \rightarrow X$, where $G$ is a convex subset of $X$. For the inclusions

$$
T x_{n}+C x_{n}+\alpha_{n} x_{n} \ni p_{n}, \quad n=1,2, \ldots,
$$

assume the following:

(a) $\left\{x_{n}\right\} \subset D(T) \cap \bar{G}, x_{n} \rightarrow x_{0} \in \overline{D(T)}$ and $C x_{n} \rightarrow C x_{0}$;

(b) $\left\{\alpha_{n}\right\} \subset R_{+}$and $\alpha_{n} \rightarrow 0$;

(c) $\left\{p_{n}\right\} \subset X$ and $p_{n} \rightarrow p \in X$.

Then $x_{0} \in D(T) \cap \bar{G}$ and $T x_{0}+C x_{0} \ni p$.

Proof. The proof follows from that of Lemma 1 in [15], where there is no set $G$. All that we need to do in order to state Lemma 1 in [15] is replace $G$ above by $D(T)$. .

We recall that since $X$ is uniformly convex, $\overline{D(T)}$ is a convex set. This can be found in Barbu [2, Proposition 3.6] and Cioranescu [7, Theorem 1.15, p. 184]. Both authors assumed that $X^{*}$ is also uniformly convex, but this is not needed in the proof. Actually Reich has shown in [36] that $\overline{D(T)}$ is a convex set even if $X^{*}$ has only a Fréchet differentiable norm.

Lemma 2. Let $T: X \supset D(T) \rightarrow 2^{X}$ be $m$-accretive with $(T+I)^{-1}$ compact. Let $C: \bar{G} \rightarrow X$ be continuous and bounded, where $G$ is a bounded subset of $X$. Let $\left\{\alpha_{n}\right\}$ be a sequence of positive numbers such that $\alpha_{n} \rightarrow 0$ as $n \rightarrow \infty$. Let $\left\{x_{n}\right\} \subset D(T) \cap \bar{G}$ and $\left\{p_{n}\right\} \subset X$ be such that $p_{n} \rightarrow p$ as $n \rightarrow \infty$ and

$$
T x_{n}+C x_{n}+\alpha_{n} x_{n} \ni p_{n}, \quad n=1,2, \ldots
$$

Then $\left\{x_{n}\right\}$ contains a subsequence $\left\{x_{n_{k}}\right\}$ such that $x_{n_{k}} \rightarrow x_{0} \in D(T) \cap \bar{G}$ and

$$
T x_{0}+C x_{0} \ni p .
$$

Proof. Let $\left\{x_{n}\right\},\left\{a_{n}\right\},\left\{p_{n}\right\}$ be as in the statement of the lemma. Then we have

$$
T x_{n}+C x_{n}+\alpha_{n} x_{n}+x_{n} \ni p_{n}+x_{n},
$$

or

$$
x_{n}=(I+T)^{-1}\left[-\left(C x_{n}+\left(\alpha_{n}-1\right) x_{n}-p_{n}\right)\right] .
$$

Since $(I+T)^{-1}$ is compact and $\left\{x_{n}\right\}$ and $C$ are bounded, we have that $\left\{x_{n}\right\}$ lies in a compact set. As such, it has a convergent subsequence $\left\{x_{n_{k}}\right\}$. Let $x_{n_{k}} \rightarrow x_{0} \in$ $\bar{G}$. Then, for some sequence of terms $v_{n_{k}} \in T x_{n_{k}}$, we have

$$
v_{n_{k}} \rightarrow-C x_{0}+p \text { as } k \rightarrow \infty .
$$

Since $T$ is $m$-accretive, it is closed. Thus, $x_{0} \in D(T)$ and $T x_{0}+C x_{0} \ni p$.

Our first theorem is one of the main results in this paper.

Theorem 1. Let $G$ be a bounded, open and convex subset of $X$. Let $T: X \supset$ $D(T) \rightarrow 2^{X}$ be $m$-accretive and (i) $C: \bar{G} \rightarrow X$ compact. Assume that $D(T) \cap G \neq \emptyset$ and that $[I-(T+C)](D(T) \cap \partial G) \subset \bar{G}$. Then $0 \in \overline{R(T+C)}$. Assume that one of the following conditions holds:

(a) $X$ is uniformly convex and $C$ is completely continuous;

(b) instead of (i), let $J_{1}$ be compact and $C: \bar{G} \rightarrow X$ continuous and bounded. Then $0 \in R(T+C)$. 
Proof. We may assume that $0 \in D(T) \cap G$ and $0 \in T(0)$. In fact, if one of these is not true, we may consider instead the operators $\widetilde{T}, \widetilde{C}$ defined on $D(\widetilde{T})$ and $\overline{\widetilde{G}}$, respectively, where $D(\widetilde{T}) \equiv D(T)-x_{0}, \widetilde{G} \equiv G-x_{0}, \widetilde{T} x \equiv T\left(x+x_{0}\right)-v_{0}$ and $\widetilde{C} x \equiv C\left(x+x_{0}\right)+v_{0}$. Here, $x_{0}$ is a fixed point in $D(T) \cap G$ and $v_{0}$ is a fixed point in $T x_{0}$. It is easy to see that our assumptions are still satisfied with these arrangements.

We are going to show first that $0 \in R(T+C+\epsilon I)$, i.e., we are going to solve first the inclusion

$$
T x+C x+\epsilon x \ni 0
$$

in $D(T) \cap \bar{G}$, for each $\epsilon>0$. We fix $\epsilon>0$ and consider the homotopy inclusion

$$
t(T+C) x+[1-t(1-\epsilon)] x \ni 0 .
$$

This inclusion gives rise to the homotopy mapping

$$
H(t, x) \equiv[t(T+\epsilon I)+(1-t) I]^{-1}(-t C x), \quad(t, x) \in[0,1] \times \bar{G} .
$$

We observe that $H(0, x) \equiv 0$. We may assume that $x-H(1, x)=0$ has no solutions $x \in \partial G$. In fact, if this is not true, then we have a solution of (2) and the proof is finished. The mapping $H(t, \cdot)$ is continuous on $\bar{G}$ and maps $\bar{G}$ onto a relatively compact subset of $X$, for every $t \in[0,1]$. The Leray-Schauder degree $d(I-H(t, \cdot), G, 0), t \in[0,1]$, will be well-defined if we show that $(\mathrm{j}) H(\cdot, x)$ is continuous uniformly w.r.t. $x \in \bar{G}$, and (jj) the equation $x-H(t, x)=0$ has no solutions $x_{t} \in \partial G$, for any $t \in[0,1)$. In order to show $(\mathrm{j})$, we set

$$
\lambda(t) \equiv t /[\epsilon t+(1-t)]
$$

and we observe that $\lambda(t), t \in[0,1]$, is a well-defined continuous function. We also observe that

$$
H(t, x)=(\lambda(t) T+I)^{-1}(-\lambda(t) C x)=J_{\lambda(t)}(-\lambda(t) C x) .
$$

Since $J_{\lambda(t)}(0)=0$ and $J_{\lambda}(t)$ is a nonexpansive mapping,

$$
\left\|J_{\lambda(t)}(-\lambda(t) C x)\right\| \leq\|\lambda(t) C x\|=\lambda(t)\|C x\| .
$$

Since $\lambda(0)=0$, we have the continuity of $J_{\lambda(t)}(-\lambda(t) C x)$ w.r.t. $t$ at $t=0$. Since $C$ is bounded, this continuity is uniform w.r.t. $x \in \bar{G}$.

Now, we fix $t, t_{0} \in(0,1], u \in X$ and let $y(t) \equiv J_{\lambda(t)} u$. Since

$$
T_{\lambda(t)} u=(1 / \lambda(t))\left(I-J_{\lambda(t)}\right) u,
$$

we have

$$
y(t)=u-\lambda(t) T_{\lambda(t)} u .
$$


Working as in the proof of Theorem 3 in [21], we obtain

$$
\left\|y(t)-y\left(t_{0}\right)\right\| \leq \frac{2\left|\lambda(t)-\lambda\left(t_{0}\right)\right|}{\lambda\left(t_{0}\right)}\|u\| .
$$

Consequently,

$$
\begin{aligned}
\| J_{\lambda(t)}(-\lambda(t) C x)-J_{\lambda\left(t_{0}\right)} & \left.-\lambda\left(t_{0}\right) C x\right) \| \\
\leq & \left\|J_{\lambda(t)}(-\lambda(t) C x)-J_{\lambda\left(t_{0}\right)}(-\lambda(t) C x)\right\| \\
& +\left\|J_{\lambda\left(t_{0}\right)}(-\lambda(t) C x)-J_{\lambda\left(t_{0}\right)}\left(-\lambda\left(t_{0}\right) C x\right)\right\| \\
\leq & \frac{2\left|\lambda(t)-\lambda\left(t_{0}\right)\right| \lambda(t)}{\lambda\left(t_{0}\right)}\|C x\|+\left|\lambda(t)-\lambda\left(t_{0}\right)\right|\|C x\| \\
= & \frac{\left(2 \lambda(t)+\lambda\left(t_{0}\right)\right)\left|\lambda(t)-\lambda\left(t_{0}\right)\right|}{\lambda\left(t_{0}\right)}\|C x\|,
\end{aligned}
$$

which shows the continuity of $H(t, x)$, w.r.t. $t$ at $t_{0}$, uniformly w.r.t. $x \in \bar{G}$. A simple covering argument shows now the desired uniform continuity of $H(t, x)$ w.r.t. $t \in[0,1]$.

To show $(\mathrm{jj})$, assume that $x_{t}=H\left(t, x_{t}\right)$, for some $t \in[0,1)$ and some $x_{t} \in$ $\partial G$. Since $t=0$ is impossible, because $x_{t} \in \partial G$ implies $x_{t} \neq 0$, we have $t \in(0,1)$. Also,

$$
t(T+C+\epsilon I) x_{t}+(1-t) x_{t} \ni 0 .
$$

Let $S x \equiv x-(T+C) x$. Then $x_{t} \in[t /(1+\epsilon t)] S x_{t}$. By our assumption, $S x_{t} \subset \bar{G}$. Since every element of $[t /(1+\epsilon t)] S x_{t}$ is a proper convex combination of $0 \in G$ and some element of $S x_{t}$, we have $[t /(1+\epsilon t)] S x_{t} \subset G$, i.e., a contradiction to the fact that $x_{t} \in \partial G$. It follows that

$$
d(I-H(t, \cdot), G, 0)=d(I-H(0, \cdot), G, 0)=1
$$

and, by the Leray-Schauder theory, the equation $x-H(1, x)=0$ is solvable in $G$. This says that the inclusion (2) is solvable in $D(T) \cap G$. Letting $x_{\epsilon}$ denote a solution of (2), and then letting $\epsilon \rightarrow 0$ ( $\left\{x_{\epsilon}\right\}$ is bounded), we have that $0 \in$ $\overline{R(T+C)}$.

If we assume, in addition, that $X$ is uniformly convex and $C$ is completely continuous, then $C$ is compact (by the reflexivity of $X$ ) and $0 \in R(T+C$ ) by Lemma 1. In fact, for some positive sequence $\left\{\epsilon_{n}\right\}$, with $\epsilon_{n} \downarrow 0$, we may take, in Lemma $1, x_{n}=x_{\epsilon_{n}}, \alpha_{n}=\epsilon_{n}$ and $p_{n}=0, n=1,2, \ldots$. Since $\left\{x_{n}\right\}$ is bounded and $X$ is reflexive, we may assume that $x_{n} \rightarrow x_{0} \in \bar{G}$.

On the other hand, in case (b) we observe that the resolvent identity

$$
J_{\mu} x=J_{\lambda}\left(\frac{\lambda}{\mu} x+\frac{\mu-\lambda}{\mu} J_{\mu} x\right)
$$

implies that $J_{\mu}$ is compact for every $\mu>0$. Thus, the entire proof of the first conclusion of the theorem goes through with no change. We let $\epsilon_{n} \downarrow 0$ and apply Lemma 2. The proof is complete.

We are now ready for a new fixed point theorem involving completely continuous perturbations of possibly discontinuous $m$-dissipative operators. 
Corollary 1. Let $G$ be an open, bounded and convex subset of $X$. Let $T: X \supset$ $D(T) \rightarrow 2^{X}$ be $m$-dissipative and $C: \bar{G} \rightarrow X$. Assume that $D(T) \cap G \neq \emptyset$ and $(T+C)(D(T) \cap \partial G) \subset \bar{G}$. Then if (a) or (b) of Theorem 1 holds, the operator $T+C$ has a fixed point in $D(T) \cap \bar{G}$, i.e., there exists $x \in D(T) \cap \bar{G}$ such that $(T+C) x \ni x$.

Proof. The operator $I-T$ is $m$-accretive. Also, the operator $I-(I-(T+C))=T+C$ maps $D(T) \cap \partial G$ into $\bar{G}$. Theorem 1 (with $I-T$ in place of $T$ and $-C$ in place of $C)$ implies that the operator $I-(T+C)$ has a zero in $D(T) \cap \bar{G}$. This completes the proof.

Corollary 1 is an extension of Theorem (13.24) of Browder [5]. The homotopy used in Theorem 1 can be modified so that it can accommodate a new result on the surjectivity of $T+C$ based on an "inner product" condition. The next theorem reflects this situation and extends to the present case a host of surjectivity results in the references. We let $\Gamma$ denote the family of all functions $\beta: R_{+} \rightarrow R_{+}$such that $\beta(\rho) \rightarrow 0$ as $\rho \rightarrow \infty$. Sometimes, the functions $\beta(\rho)$ considered in this paper are only defined for all sufficiently large $\rho>0$. We make no comment on their trivial definition on any initial finite interval in $\mathcal{R}_{+}$. For a set $A \subset X$, we set $|A| \equiv \inf \{\|x\| ; x \in A\}$. We denote by $\operatorname{co} G$ the convex hull of the set $G$.

Theorem 2. Let $T: X \supset D(T) \rightarrow 2^{X}$ be $m$-accretive and (i) $C: D(T) \rightarrow X$ is compact. Assume that there exist $\beta \in \Gamma$ and $Q>0$ such that: for every $x \in D(T)$ with $\|x\| \geq Q$ and every $v \in T x$ there exists $j \in J x$ such that

$$
\langle v+C x, j\rangle \geq-\beta(\|x\|)\|x\|^{2} .
$$

Then $R(T+C+\epsilon I)=X$, for every $\epsilon \in(0,1)$. Assume, further, that

$$
\liminf _{\substack{\|x\| \rightarrow \infty \\ x \in D(T)}} \frac{|T x+C x|}{\|x\|}>0 .
$$

Then $\overline{R(T+C)}=X$. Let one of the following conditions hold:

(a) $X$ is uniformly convex and $C: \overline{D(T)} \rightarrow X$ is completely continuous;

(b) instead of (i), $C: \overline{D(T)} \rightarrow X$ is continuous and bounded and $(T+I)^{-1}$ is compact.

Then $R(T+C)=X$.

Proof. We are planning to solve first the problem

$$
T x+C x+\epsilon x \ni s,
$$

where $s$ is any (but fixed) point in $X$. We may assume that, for some $x_{0} \in D(T)$, we have $0 \in T\left(x_{0}\right)$. If this is not true, we consider instead the operators $\widetilde{T}(x) \equiv$ $T x-v_{0}, \widetilde{C}(x) \equiv C x+v_{0}$, for every $x \in D(T)$, where $v_{0}$ is any (but fixed) point in $T x_{0}$. We assume that (i) holds in order to prove the first conclusion of the theorem. In order to solve (4), we consider the homotopy inclusion

$$
t(T x+C x+\epsilon x)+(1-t) x \ni t s,
$$


or the equation

$$
\begin{aligned}
u=H(t, u) & \left.\equiv-t\left[C(t(T+\epsilon I)+(1-t) I)^{-1} u-s\right)\right] \\
& =-t\left[C((t / \lambda(t)) T+I)^{-1}([1 / \lambda(t)] u)-s\right],
\end{aligned}
$$

where we have set $H(0, x) \equiv 0$ and $\lambda(t) \equiv[1-t(1-\epsilon)]$. It is easy to see that $\epsilon \leq \lambda(t) \leq 1, t \in[0,1]$. For every solution $u \in X$ of $u-H(1, u)=0$, we obtain a solution $x$ of $(4)$, where $x=(T+\epsilon I)^{-1} u$. In order to apply the Leray-Schauder degree theory, we shall show first that the mapping $H:[0,1] \times \overline{B_{r}(0)} \rightarrow X$ is continuous and such that $H\left([0,1], \overline{B_{r}(0)}\right)$ is relatively compact in $X$, for every $r>0$. To this end, we fix $r>0$ and show that the function

$$
y(t, u) \equiv((t / \lambda(t)) T+I)^{-1}([1 / \lambda(t)] u)
$$

is continuous in $(t, u) \in(0,1] \times \overline{B_{r}(0)}$. In fact, setting $\mu(t) \equiv t / \lambda(t), q(t) \equiv 1 / \lambda(t)$ and taking into consideration the proof of Theorem 1 , we obtain

$$
\begin{aligned}
\left\|y(t, u)-y\left(t_{0}, v\right)\right\|= & \left\|J_{\mu(t)} q(t) u-J_{\mu\left(t_{0}\right)} q\left(t_{0}\right) v\right\| \\
\leq & \left\|J_{\mu(t)} q(t) u-J_{\mu\left(t_{0}\right)} q(t) u\right\| \\
& +\left\|J_{\mu\left(t_{0}\right)} q(t) u-J_{\mu\left(t_{0}\right)} q\left(t_{0}\right) v\right\| \\
\leq & \frac{2\left|\mu(t)-\mu\left(t_{0}\right)\right|}{\mu\left(t_{0}\right)}\|q(t) u\| \\
& +\left\|q(t) u-q\left(t_{0}\right) v\right\| .
\end{aligned}
$$

The continuity of $y(t, u)$ on $(0,1] \times \overline{B_{r}(0)}$ follows from this inequality because the functions $\mu(t), \lambda(t)$ and $q(t)$ are well-defined, continuous and bounded on the interval $(0,1]$. Since $C$ is continuous, the continuity of $H(t, u)=-t[C y(t, u)-s]$ on $(0,1] \times \overline{B_{r}(0)}$ has been shown. In order to show the continuity of $H(t, u)$ on the set $\{0\} \times \overline{B_{r}(0)}$, we observe first that

$$
\begin{aligned}
\|y(t, u)\|=\left\|J_{\mu(t)} q(t) u\right\| & \leq\left\|J_{\mu(t)} q(t) u-J_{\mu(t)} x_{0}\right\|+\left\|J_{\mu(t)} x_{0}\right\| \\
& \leq\left\|q(t) u-x_{0}\right\|+\left\|x_{0}\right\| \\
& \leq r \sup _{t \in[0,1]} q(t)+2\left\|x_{0}\right\| .
\end{aligned}
$$

This implies that the set

$$
K=\left\{y(t, u):(t, u) \in[0,1] \times \overline{B_{r}(0)}\right\}(\subset D(T))
$$

is bounded. Hence, from the boundedness of $C$ we obtain that

$$
\|H(t, u)\| \leq t\left\|C J_{\mu(t)} q(t) u-s\right\| \rightarrow 0 \text { as } t \rightarrow 0,
$$

uniformly w.r.t. $u \in \overline{B_{r}(0)}$. We have demonstrated the continuity of the homotopy function $H(t, u)$ on the set $[0,1] \times \overline{B_{r}(0)}$. 
To show that the set $H\left([0,1] \times \overline{B_{r}(0)}\right)$ is relatively compact, let $\left(t_{n}, u_{n}\right)$ be a sequence of points in $[0,1] \times \overline{B_{r}(0)}$. We are going to show that the sequence of points

$$
z\left(t_{n}, u_{n}\right) \equiv-t_{n}\left[C y\left(t_{n}, u_{n}\right)-s\right]
$$

contains a convergent subsequence. To this end, we observe first that since $[0,1]$ is compact, we may assume that $t_{n} \rightarrow t_{0}$, for some $t_{0} \in[0,1]$. If $t_{0}=0$, then the boundedness of the sequence $\left\{C y\left(t_{n}, u_{n}\right)-s\right\}$ implies that $z\left(t_{n}, u_{n}\right) \rightarrow 0$. Thus, we may assume that $t_{0}>0$ and $t_{n}>0$ for all $n$. We are now going to show the existence of a convergent subsequence of the sequence $\left\{y\left(t_{n}, u_{n}\right)\right\}$. If $C$ is compact, then the continuity of $C: D(T) \rightarrow X$ implies immediately the existence of such a convergent subsequence because, by the continuity of multiplication and translation, the set $-[0,1](\overline{C K}-s)$ is compact and $z\left(t_{n}, u_{n}\right)$ lies in this set. If $(T+I)^{-1}$ is compact, then the resolvent identity says that $\left(\mu\left(t_{0}\right) T+I\right)^{-1}$ is also compact. From the definition of $y\left(t_{n}, u_{n}\right)$ we have

$$
\mu\left(t_{n}\right) T y\left(t_{n}, u_{n}\right)+y\left(t_{n}, u_{n}\right) \ni q\left(t_{n}\right) u_{n},
$$

or

$$
\mu\left(t_{n}\right) v_{n}+y\left(t_{n}, u_{n}\right)=q\left(t_{n}\right) u_{n},
$$

where $v_{n} \in T y\left(t_{n}, u_{n}\right)$. This implies

$$
\mu\left(t_{0}\right) v_{n}+y\left(t_{n}, u_{n}\right)=q\left(t_{n}\right) u_{n}-\left[\mu\left(t_{n}\right)-\mu\left(t_{0}\right)\right] v_{n},
$$

or

$$
\begin{aligned}
y\left(t_{n}, u_{n}\right) & =\left(\mu\left(t_{0}\right) T+I\right)^{-1}\left[q\left(t_{n}\right) u_{n}-\left[\mu\left(t_{n}\right)-\mu\left(t_{0}\right)\right] v_{n}\right] \\
& =\left(\mu\left(t_{0}\right) T+I\right)^{-1}\left[q\left(t_{n}\right) u_{n}-\frac{\left[\mu\left(t_{n}\right)-\mu\left(t_{0}\right)\right]\left[q\left(t_{n}\right) u_{n}-y\left(t_{n}, u_{n}\right)\right]}{\mu\left(t_{n}\right)}\right] .
\end{aligned}
$$

The compactness of the resolvent $J_{\mu\left(t_{0}\right)}$ and the boundedness of the sequence of points to the right of it above $\left(\mu\left(t_{n}\right) \rightarrow t_{0} / \lambda\left(t_{0}\right)>0\right)$ show that the sequence $\left\{y\left(t_{n}, u_{n}\right)\right\}$ lies in a compact set. It thus has a convergent subsequence. Since $C$ is continuous, we obtain a convergent subsequence of the sequence $\left\{z\left(t_{n}, u_{n}\right)\right\}$.

In order to show that $d(I-H(t, \cdot), B, 0)=$ const., for some open ball $B \subset X$, we are going to show first that all possible solutions $u$ of the equation $u-H(t, u)=0$ are bounded by a constant which is independent of the parameter $t \in[0,1]$. To this end, let us assume that $\left\{u_{m}\right\}$ is a sequence of points in $X$ such that $u_{m}-H\left(t_{m}, u_{m}\right)=0$, for some sequence $\left\{t_{m}\right\} \subset[0,1]$, and $\left\|u_{m}\right\| \rightarrow \infty$ as $m \rightarrow \infty$. Let

$$
x_{m}=\left(t_{m}(T+\epsilon I)+\left(1-t_{m}\right) I\right)^{-1} u_{m} .
$$

Then we have $\left\{x_{m}\right\} \subset D(T)$ and

$$
t_{m}(T+\epsilon I) x_{m}+t_{m}\left(C x_{m}-s\right)+\left(1-t_{m}\right) x_{m} \ni 0,
$$

or

$$
t_{m} v_{m}+t_{m}\left(C x_{m}-s\right)+\left[1-t_{m}(1-\epsilon)\right] x_{m}=0,
$$


for some $v_{m} \in T x_{m}$. We must have $\left\|x_{m}\right\| \rightarrow \infty$ as $m \rightarrow \infty$. In fact, if this is not true, then there exists a subsequence of $\left\{x_{m}\right\}$, denoted again for convenience by $\left\{x_{m}\right\}$, which is bounded. Then the boundedness of the operator $C$ and (6) imply that the sequence $\left\{t_{m} v_{m}\right\}$ is bounded. Since

$$
u_{m}=t_{m} v_{m}+\left[1-t_{m}(1-\epsilon)\right] x_{m},
$$

we have that $\left\{u_{m}\right\}$ is also bounded, i.e., a contradiction.

Using our hypotheses, there exist $\beta \in \Gamma$ and $Q>0$ such that: for every $m \geq 1$ such that $\left\|x_{m}\right\| \geq Q$ there exists $j \in J x_{m}$ such that

$$
\begin{aligned}
\left\langle v_{m}+C x_{m}-s, j\right\rangle & \geq-\langle s, j\rangle-\beta\left(\left\|x_{m}\right\|\right)\left\|x_{m}\right\|^{2} \\
& \geq-\frac{\|s\|}{\left\|x_{m}\right\|}\left\|x_{m}\right\|^{2}-\beta\left(\left\|x_{m}\right\|\right)\left\|x_{m}\right\|^{2} \\
& =-\widetilde{\beta}\left(\left\|x_{n}\right\|\right)\left\|x_{m}\right\|^{2},
\end{aligned}
$$

for every sequence $\left\{v_{m}\right\}$ of terms $v_{m} \in T x_{m}$, where $\widetilde{\beta}$ is an obvious function in $\Gamma$. For such an integer $m$, we evaluate the above functional $j \in J x_{m}$ on both sides of (6) in order to obtain

$$
\begin{aligned}
\epsilon\left\|x_{m}\right\|^{2} & \leq\left[1-t_{m}(1-\epsilon)\right]\left\|x_{m}\right\|^{2} \\
& \leq-t_{m}\left\langle v_{m}+C x_{m}-s, j\right\rangle \\
& \leq t_{m} \widetilde{\beta}\left(\left\|x_{m}\right\|\right)\left\|x_{m}\right\|^{2} .
\end{aligned}
$$

We see now that this last inequality holds for all large integers $m$. It implies

$$
\epsilon \leq \widetilde{\beta}\left(\left\|x_{m}\right\|\right) .
$$

Since the right-hand side above converges to zero as $m \rightarrow \infty$, we have a contradiction. We have shown that all possible solutions of $x-H(t, x)=0$ lie in an open ball, say, $B_{M}(0)$, whose radius $M>0$ is independent of the parameter $t$. It follows that $x-H(t, x) \neq 0$, for any $(t, x) \in[0,1] \times \partial B_{M}(0)$. Thus, the Leray-Schauder degree $d\left(I-H(t, \cdot), B_{M}(0), 0\right)$ is well-defined and constant for $t \in[0,1]$. In particular,

$$
\begin{aligned}
d\left(I-H(t, \cdot), B_{M}(0), 0\right) & =d\left(I-H(0, \cdot), B_{M}(0), 0\right) \\
& =d\left(I, B_{M}(0), 0\right) \\
& =1
\end{aligned}
$$

because $0 \in B_{M}(0)$. We conclude that the equation $x-H(1, x)=0$ has a solution $x \in B_{M}(0)$. This solution satisfies the inclusion (5). It follows that $s \in R(T+C+\epsilon I)$, for every $\epsilon \in(0,1)$.

For the second part of the proof, we let $x_{n}$ be a solution to the problem (4) for $\epsilon=1 / n$. We claim that $\left\{x_{n}\right\}$ is a bounded sequence. To see this, assume, without loss of generality, that $\left\|x_{n}\right\| \rightarrow \infty$ as $n \rightarrow \infty$. Then, by our hypothesis, there exists $\alpha>0$ so that

$$
\liminf _{n \rightarrow \infty} \frac{\left|T x_{n}+C x_{n}\right|}{\left\|x_{n}\right\|} \geq \liminf _{\substack{\|x\| \rightarrow \infty \\ x \in D(T)}} \frac{|T x+C x|}{\|x\|}=\alpha .
$$


However, we know that for some $v_{n} \in T x_{n}$ we have

$$
\left\|v_{n}+C x_{n}\right\|=\left\|s-(1 / n) x_{n}\right\| \leq(1 / n)\left\|x_{n}\right\|+\|s\|,
$$

which implies

$$
\alpha \leq \liminf _{n \rightarrow \infty} \frac{\left\|v_{n}+C x_{n}\right\|}{\left\|x_{n}\right\|} \leq \liminf _{n \rightarrow \infty}\left[(1 / n)+\frac{\|s\|}{\left\|x_{n}\right\|}\right]=0,
$$

i.e., a contradiction. Since $(1 / n) x_{n} \rightarrow 0$, we have that $s \in \overline{R(T+C)}$.

If (a) is true, then since $\left\{x_{n}\right\}$ is a bounded sequence, we may assume that $x_{n} \rightarrow x_{0} \in \overline{D(T)} \cap \overline{\operatorname{co}\left\{x_{n}\right\}}$, and then apply Lemma 1 with $G=\operatorname{co}\left\{x_{n}\right\}$.

If (b) is true, then we apply Lemma 2 with $G$ any ball $B_{r}(0)$ of $X$ containing $\left\{x_{n}\right\}$.

We note that (3) is an extremely weak boundary condition and that condition (*) in the statement of Theorem 2 does not generally suffice for $\overline{R(T+C)}=X$ even if $T: X \rightarrow X$ is continuous. In fact, let $T x \equiv x^{m}$, for some odd positive integer $m, C x \equiv|x|^{m}+|x|+3$ and $\alpha \in(0,1)$. Then $T: \mathcal{R} \rightarrow \mathcal{R}$ is $m$-accretive, $C: \mathcal{R} \rightarrow \mathcal{R}$ is compact and

$$
\liminf _{|x| \rightarrow \infty} \frac{|T x+C x|}{|x|} \geq 1
$$

but $0 \notin \overline{R(T+C)}=[3, \infty)$. For more uses of boundary conditions like (but different from) (3), the reader is referred to the paper [17].

On the other hand, condition (3) is not generally sufficient for $\overline{R(T+C)}=X$ either. In fact, if $T x \equiv x^{1 / 3}$ and $C x \equiv|x|^{1 / 3}+3$, then we have

$$
\begin{aligned}
\langle T x+C x, x\rangle & =(T x+C x) x \geq-|x|^{4 / 3}-3|x| \\
& =-\left(1 /|x|^{2 / 3}\right)|x|^{2}-(3 /|x|)|x|^{2}=-\beta_{0}(|x|)|x|^{2},
\end{aligned}
$$

for all $x \neq 0$, where $\beta_{0} \in \Gamma$. However, $0 \notin \overline{R(T+C)}=[3, \infty)$.

It is easy to see that (3) is implied by any other condition which has the same left-hand side while its right-hand side is of the form

$$
-K-L\|x\|-\beta(\|x\|)\|x\|^{2},
$$

for some positive constants $K, L$. In fact,

$$
\begin{aligned}
-K-L\|x\|-\beta(\|x\|)\|x\|^{2} & =-\frac{K}{\|x\|^{2}}\|x\|^{2}-\frac{L}{\|x\|}\|x\|^{2}-\beta(\|x\|)\|x\|^{2} \\
& =-\widetilde{\beta}(\|x\|)\|x\|^{2}
\end{aligned}
$$

for all $x \in D(T)$ with $\|x\| \geq Q$, where $\widetilde{\beta}$ is an obvious function in $\Gamma$.

The next result provides a method for proving the existence of certain eigenvalues for the pair $(T, C)$. It is assumed that $C J_{\lambda}$ is at least condensing, for some $\lambda>0$, and shown that $\lambda+1$ is an eigenvalue for $(T, C)$. A real number $\lambda$ is called an "eigenvalue" of a pair of operators $(T, C)$ if the equation $\lambda T x+C x \ni 0$ is solvable in $D(T) \cap D(C)$. 
Theorem 3. Let $G$ be a bounded, open and convex subset of $X$ and let $T: X \supset$ $D(T) \rightarrow 2^{X}$ be $m$-accretive with $0 \in D(T) \cap G$ and $0 \in T(0)$. Assume that $C$ : $D(T) \rightarrow X$ is bounded and such that (i) $C(\lambda T+I)^{-1}$ is compact for some $\lambda>0$. Let $[I-(T+C)](D(T) \cap \partial G) \subset \bar{G}$ and $[(\lambda T+I)(D(T) \cap \partial G)] \cap \operatorname{int} G=\emptyset$. Then $0 \in \overline{R((\lambda+1) T+C)}$. Assume that one of the following conditions holds:

(a) $X$ is uniformly convex and $C(\lambda T+I)^{-1}$ is completely continuous;

(b) instead of (i), $J_{1}$ is compact and $C(\lambda T+I)^{-1}$ is just condensing.

Then the number $\lambda+1$ is an eigenvalue for the pair $(T, C)$.

Proof. We consider the homotopy equation

$$
t\left(T_{\lambda}+C J_{\lambda}\right) x+(1+\epsilon) x-t J_{\lambda} x=0 .
$$

Here, $t \in[0,1], \epsilon>0$ and $x \in \bar{U}$, where $U \equiv(\lambda T+I)(D(T) \cap G)$. We are planning to solve first the equation

$$
T_{\lambda} x+C J_{\lambda} x+(1+\epsilon) x-J_{\lambda} x=0,
$$

which can also be written as

$$
(\lambda+1) T_{\lambda} x+C J_{\lambda} x+\epsilon x=0
$$

because $T_{\lambda}=(1 / \lambda)\left(I-J_{\lambda}\right)$. We write $(8)$ as

$$
x=\frac{t(\lambda+1)}{t+\lambda(1+\epsilon)} J_{\lambda} x-\frac{t \lambda}{t+\lambda(1+\epsilon)} C J_{\lambda} x .
$$

Since $J_{\lambda} \equiv(\lambda T+I)^{-1}$ is defined and continuous on the entire space $X$, the (possibly multi-valued) mapping $V: x \rightarrow(\lambda T+I) x$ maps relatively open (closed) sets of its domain $D(T)$ onto open (closed) subsets of $X$. Thus, $V(D(T) \cap G)$ is open and $V(D(T) \cap \bar{G})$ is closed in $X$. This implies, as in [28, Theorem 2], that

$$
\overline{(\lambda T+I)(D(T) \cap G)} \subset(\lambda T+I)(D(T) \cap \bar{G})
$$

and

$$
\partial[(\lambda T+I)(D(T) \cap G)] \subset(\lambda T+I)(D(T) \cap \partial G),
$$

which is a crucial point of our proof. Since the function $t \rightarrow t /(t+a), t \in[0,1]$, is increasing for every $a>0$, it is evident that the first term on the right-hand side of (11) is a strict contraction for all $t \in[0,1]$. In addition, the second term is a compact mapping for all $t \in[0,1]$. Thus, the right-hand side of (11), denoted by $H(t, x)$, is a condensing mapping w.r.t. $x \in \bar{U}$ for every $t \in[0,1]$. It is also jointly continuous. As such, it is a homotopy of condensing mappings such that the image of the set $\bar{U}$ by $H(t, \cdot)$ is bounded. Thus, the degree $d(I-H(t, \cdot), U, 0)$ will be well-defined, constant and equal to 1 , if the equation $x-H(t, x)=0$ has no solution $x_{t}$ on the boundary of the open set $U$, for any $t \in[0,1]$. The proof of the existence of such a degree is sketched in Theorem 1 of [28]. Let $x_{t} \in \partial U$ be a solution of this equation for some $t \in[0,1]$. Then

$$
t\left(T_{\lambda}+C J_{\lambda}\right) x_{t}+(1+\epsilon) x_{t}-t J_{\lambda} x_{t}=0 .
$$


This implies

$$
x_{t}=\frac{t}{1+\epsilon}\left[J_{\lambda}-\left(T_{\lambda}+C J_{\lambda}\right)\right] x_{t} .
$$

From what we have seen above, since $x_{t} \in \partial U$, we must have $J_{\lambda} x_{t} \in D(T) \cap \partial G$. By our assumption, this implies

$$
\left[J_{\lambda}-\left(T_{\lambda}+C J_{\lambda}\right)\right] x_{t} \subset\left[J_{\lambda}-\left(T J_{\lambda}+C J_{\lambda}\right)\right] x_{t} \subset \bar{G} .
$$

Again, since $G$ is open and convex and $0 \in G$, inclusions (13) and (14) imply $x_{t} \in \operatorname{int} G$. Since we also have

$$
x_{t} \in \partial[(\lambda T+I)(D(T) \cap G)] \subset(\lambda T+I)(D(T) \cap \partial G),
$$

we get a contradiction to

$$
[(\lambda T+I)(D(T) \cap \partial G)] \cap \operatorname{int} G=\emptyset .
$$

It follows that equation (10) has a solution $x_{\epsilon} \in(\lambda T+I)(D(T) \cap G)$, for every $\epsilon>0$.

We now observe that (9) implies that the family $\left\{x_{\epsilon}\right\}$ of solutions of (10) obtained as above is bounded. In fact, each $x_{\epsilon}$ satisfies the equation (11) for $t=1$. Since $x_{\epsilon}$ lies in the set $\overline{(\lambda T+I)(D(T) \cap G)} \subset(\lambda T+I)(D(T) \cap \bar{G})$, we have that $J_{\lambda} x_{\epsilon}$ lies in the set $D(T) \cap \bar{G}$. Since this set is bounded and $C$ is also bounded, equation (11) (for $t=1$ ) implies that $\left\{x_{\epsilon}\right\}$ lies in a bounded set. Thus, $\epsilon x_{\epsilon} \rightarrow 0$ and $0 \in$ $\overline{R((\lambda+1) T+C)}$.

For the second part of the theorem, we let $\epsilon=1 / n$ and $x_{n} \equiv x_{1 / n}$, and observe that since $X$ is reflexive, there exists a subsequence of $\left\{x_{n}\right\}$, denoted again by $\left\{x_{n}\right\}$, such that $x_{n} \rightarrow x \in X$. Since $C J_{\lambda}$ is completely continuous, we have that $C J_{\lambda} x_{n} \rightarrow C J_{\lambda} x$. Thus, we may apply Lemma 1 with $T_{\lambda}$ in place of $T, C J_{\lambda}$ in place of $C$ and $X$ in place of $D(T)$ or $G$.

In the third part of the proof, we observe first that since the operator $J_{\lambda}$ is compact, we do not need the term $\epsilon x$ in the equation (9). Because of this, the homotopy equation (11) must now be replaced by the equation

$$
x=\frac{t(\lambda+1)}{t+\lambda} J_{\lambda} x-\frac{t \lambda}{t+\lambda} C J_{\lambda} x .
$$

We observe that the first term of this equation is compact w.r.t. $x$ and the second condensing, for every $t \in[0,1]$. We may repeat now the previous argument in order to solve the equation (9) with zero in place of $\epsilon$. This immediately implies $\left((\lambda+1) T J_{\lambda}+C J_{\lambda}\right) x \ni 0$ and finishes the proof.

A special, and important, case of Theorem 2 occurs when $G=B_{r}(0)$, for some $r>0$. This is the content of Corollary 2 .

Corollary 2. Let $T: X \supset D(T) \rightarrow 2^{X}$ be $m$-accretive with $0 \in D(T)$ and $0 \in$ $T(0)$. Assume that $C: D(T) \rightarrow X$ is bounded and such that (i) $C(\lambda T+I)^{-1}$ is compact, for some $\lambda>0$. Let $[I-(T+C)]\left(D(T) \cap \partial B_{r}(0)\right) \subset \overline{B_{r}(0)}$. Then $0 \in \overline{R((\lambda+1) T+C)}$. Assume that one of the following conditions holds:

(a) $X$ is uniformly convex and $C(\lambda T+I)^{-1}$ is completely continuous;

(b) instead of (i), $J_{1}$ is compact and $C(\lambda T+I)^{-1}$ is just condensing. 
Then the number $\lambda+1$ is an eigenvalue for the pair $(T, C)$.

Proof. In view of Theorem 3 , it suffices to show that

$$
\left[(\lambda T+I)\left(D(T) \cap \partial B_{r}(0)\right)\right] \cap B_{r}(0)=\emptyset .
$$

In fact, if $y \in(\lambda T+I)\left(D(T) \cap \partial B_{r}(0)\right)$, then $y=\lambda v+x$, where $v \in T x$, for some $x \in D(T) \cap \partial B_{r}(0)$. Since $0 \in T(0)$ and $T$ is accretive, we have, for some $j \in J x$,

$$
\|\lambda v+x\| r=\|\lambda v+x\|\|x\| \geq\langle\lambda v+x, j\rangle=\lambda\langle v, j\rangle+\langle x, j\rangle \geq\|x\|^{2}=r^{2} .
$$

This implies that $\|\lambda v+x\| \geq r$ and proves that $y \notin B_{r}(0)$.

Corollary 2 implies the existence of a zero of the operator $T+C$ if we assume that $(T+I)^{-1}$ is compact and $C: \overline{D(T)} \rightarrow X$ is continuous and bounded. In fact, these two assumptions imply that $(\lambda T+I)^{-1}$ and $C(\lambda T+I)^{-1}$ are compact due to the resolvent identity.

Corollary $2^{\prime}$. Let $T: X \supset D(T) \rightarrow 2^{X}$ be m-accretive with $0 \in D(T)$ and $0 \in$ $T(0)$. Assume that $C: \overline{D(T)} \rightarrow X$ is continuous and bounded. Let $(T+I)^{-1}$ be compact and $[I-(T+C)]\left(D(T) \cap \partial B_{r}(0)\right) \subset \overline{B_{r}(0)}$, for some $r>0$. Then, for every $\lambda \in \mathcal{R}_{+}, 0 \in R((\lambda+1) T+C)$.

Proof. We know from Corollary 2 that $0 \in R((\lambda+1) T+C)$, for all $\lambda>0$. To show this for $\lambda=0$, we note first that the solvability of $((\lambda+1) T+C) x \ni 0$, in the proof of Corollary 2, follows from the solvability of $\left((\lambda+1) T_{\lambda}+C J_{\lambda}\right) x \ni 0$, where $x \in(\lambda T+I)\left(D(T) \cap B_{r}(0)\right)$. Let $\left\{\lambda_{n}\right\} \subset(0, \infty)$ be such that $\lambda_{n} \downarrow 0$ and let $x_{n} \in(\lambda T+I)\left(D(T) \bigcap B_{r}(0)\right)$ be such that

$$
T_{\lambda_{n}} x_{n}+\left[1 /\left(\lambda_{n}+1\right)\right] C J_{\lambda_{n}} x_{n}=0 .
$$

Letting $u_{n} \equiv J_{\lambda_{n}} x_{n} \in D(T) \cap B_{r}(0)$, we have that $\left\{u_{n}\right\}$ is bounded and

$$
u_{n}=(T+I)^{-1}\left(-\left[1 /\left(\lambda_{n}+1\right)\right] C u_{n}+u_{n}\right) .
$$

Consequently, due to the boundedness of $C$ and the compactness of $(T+I)^{-1}$, we have that $u_{n} \rightarrow u_{0} \in \overline{D(T) \cap B_{r}(0)}$. Since, for some $v_{n} \in T u_{n}, v_{n} \rightarrow-C u_{0}$, the closedness of $T$ implies that $u_{0} \in D(T)$ and $T u_{0}+C u_{0} \ni 0$.

It would be very interesting to know anything about the relationship between the boundary condition

$$
[I-(T+C)]\left(D(T) \cap \partial B_{r}(0)\right) \subset \overline{B_{r}(0)}
$$

and the following "inner product" condition: for every $x \in D(T) \cap \partial B_{r}(0)$ there exists $j \in J x$ such that

$$
\langle u+C x, j\rangle \geq 0,
$$

for every $u \in T x$. This last boundary condition and other extensions of it provide the standard criteria for the existence of zeros of the operator $T+C$.

The following variant of Theorem 2 provides conditions for the existence of zeros of $T+C$. It improves Theorem 5 in [29]. 
Theorem 4. Let $G$ be a bounded, open and convex subset of $X$ and let $T: X \supset$ $D(T) \rightarrow 2^{X}$ be $m$-accretive with $0 \in D(T) \cap G$ and $0 \in T(0)$. Assume that $C$ : $D(T) \rightarrow X$ is bounded and such that (i) $C(\lambda T+I)^{-1}$ is compact for some $\lambda \in(0, \infty)$. Let $(I-\lambda C)(D(T) \cap \partial G) \subset \bar{G}$ and $[(\lambda T+I)(D(T) \cap \partial G)] \cap \operatorname{int} G=\emptyset$. Then $0 \in \overline{R(T+C)}$. Assume that one of the following conditions holds:

(a) $X$ is uniformly convex and $C(\lambda T+I)^{-1}$ is completely continuous;

(b) instead of (i), $J_{1}$ is compact, $C(\lambda T+I)^{-1}$ is just condensing and $\lambda \in(0,1]$. Then $0 \in R(T+C)$.

Proof. We proceed as in Theorem 3, but we are now using the approximate problem

$$
\left(T_{\lambda}+C J_{\lambda}\right) x+\epsilon x=0,
$$

which can be also written as

$$
(1+\epsilon \lambda) x-J_{\lambda} x+\lambda C J_{\lambda} x=0 .
$$

In order to solve (16), we consider the homotopy analogue of (11),

$$
x=H(t, x) \equiv \frac{t}{1+\epsilon \lambda} J_{\lambda} x-\frac{\lambda t}{1+\epsilon \lambda} C J_{\lambda} x,
$$

for $t \in[0,1]$ and $x \in \overline{(\lambda T+I) G}$. We note that the first term on the right-hand side of (18) is a strict contraction and the second is compact. Thus, $H(t, x)$ is condensing w.r.t. $x$. It is also jointly continuous. We can thus apply the relevant degree theory to the mapping $I-H(t, \cdot)$, as in the proof of Theorem 3. To this end, we must show that the equation $x-H(t, x)=0$ has no solution $x \in \partial((\lambda T+I)(D(T) \cap G)) \subset$ $(\lambda T+I)(D(T) \cap \partial G)$, for any $t \in[0,1]$. In fact, if $x_{t}$ were such a solution, then we would have

$$
x_{t}=\frac{t}{1+\epsilon \lambda}\left(J_{\lambda} x_{t}-\lambda C J_{\lambda} x_{t}\right) .
$$

We can now argue as in the case of equation (8) in order to obtain a contradiction. Having solved (16) for each $\epsilon>0$, we have that $0 \in \overline{R(T+C)}$.

The proof under assumption (a) follows as in Theorem 3. It is therefore omitted.

To prove the conclusion under the assumption (b), it suffices to observe that since $J_{\lambda}$ is compact, we do not need the term $\epsilon x$ in the equation (16). In fact, without this term (17) becomes

$$
x=J_{\lambda} x-\lambda C J_{\lambda} x,
$$

and the homotopy equation (18) is now replaced by

$$
x=H(t, x) \equiv t J_{\lambda} x-\lambda t C J_{\lambda} x .
$$

Since, as in the proof of Theorem $1, J_{\lambda} x$ is compact and $\lambda \in(0,1]$, the mapping $H(t, x)$ is condensing w.r.t. $x$ for every $t \in[0,1]$. If (18) has a solution $x \in$ $(\lambda T+I)(D(T) \cap \partial G)$ for $t=1$, then we are done. Since it is impossible to have such a solution for $t=0$, we may assume that $t \in(0,1)$. The solvability of $\left(T_{\lambda}+C J_{\lambda}\right) x=0$ (hence $(T+C) x \ni 0)$ now follows as above.

The following corollary sheds more light into the applicability of Theorem 3 . 
Corollary 3. Let $T: X \supset D(T) \rightarrow 2^{X}$ be $m$-accretive with $0 \in D(T)$ and $0 \in$ $T(0)$. Assume that $C: D(T) \rightarrow X$ is bounded and such that (i) $C(T+I)^{-1}$ is compact. Let

$$
\|x-C x\| \leq r, \text { for every } x \in D(T) \cap \partial B_{r}(0) .
$$

Then $0 \in \overline{R(T+C)}$. Assume, further, that one of the following conditions holds:

(a) $X$ is uniformly convex and $C(T+I)^{-1}$ is completely continuous;

(b) instead of (i), $J_{1}$ is compact and $C(T+I)^{-1}$ is just condensing. Then $0 \in R(T+C)$.

Proof. We just let $\lambda=1$ in Theorem 4 and observe that (15) holds as in the proof of Corollary 2.

As we mentioned in the introduction, Theorem A is actually true if the operator $T$ is only assumed to be continuous on $\bar{G}$. Actually, this result is true if we assume that $X^{*}$ is uniformly convex and $T$ is demicontinuous on $\bar{G}$, provided that an additional assumption is made to the effect that (i) $(I+T)(\partial G) \cap \operatorname{int} G=\emptyset$. Such an assumption is needed, as in Theorems 3 and 4 for $\lambda=1$, because the operators $J_{1}, C J_{1}$ are now defined on the set $\overline{(T+I) G}$. Due to this additional assumption, we must also assume that (ii) $0 \in G$ and $T(0)=0$. In fact, without (i), we can always assume (ii) without any loss of generality. This was shown in the proof of Theorem 1. However, unlike the continuous case, this setting cannot be handled by Browder's degree theory because, again, strongly accretive demicontinuous mappings do not necessarily belong to classes of permissible homeomorphisms. Theorem 5 below covers this case and is based upon the invariance of domain result of the author in [21]. It is easily seen that the method of proof of Theorem 1 cannot be applied here because the operator $t(T+\epsilon I)+(1-t) I$ is no longer an onto mapping and the mapping

$$
x \rightarrow[t(T+\epsilon I)+(1-t) I]^{-1}(-t C x)
$$

cannot necessarily be defined. The mapping

$$
u \rightarrow C[t(T+\epsilon I)+(1-t) I]^{-1} u
$$

cannot necessarily be handled either, because the relevant Leray-Schauder degree function would have the time-dependent domain $\overline{(t(T+\epsilon I)+(1-t) I) G}$. Such time-dependent domains were considered by Browder [5], where $T$ was at least a continuous mapping. To the best of our knowledge, no Leray-Schauder degree theory has ever been applied to time-dependent domains, as above, arising from strongly discontinuous, or even demicontinuous, operators $T$. For a degree theory involving demicontinuous operators $T$ mapping a subset of $X$ into $X^{*}$ and satisfying condition $\left(S_{+}\right)$(see Section 3), we cite the book of Skrypnik [38].

Theorem 5. Assume that $X^{*}$ is uniformly convex. Assume that $T: \bar{G} \rightarrow X$ is demicontinuous, accretive and $C: \bar{G} \rightarrow X$ compact, where $G$ is an open, convex and bounded subset of $X$ with $0 \in G$ and $T(0)=0$. Let $(T+C) \bar{G}$ be closed, $[(I+T)(\partial G)] \cap \operatorname{int} G=\emptyset$ and $[I-(T+C)](\partial G) \subset \bar{G}$. Then $(T+C) \bar{G} \ni 0$.

Proof. The proof follows as in Theorem 4. In fact, the proof is based upon a degree theoretic argument involving equation (18) with $\lambda=1$. The right-hand side of (18) again defines a condensing mapping on the set $\overline{(T+I) G}$, which is the closure of 
the open set $(T+I) G$. The set $(T+I) G$ is open by the invariance of domain result of the author in [21]. The solvability of $(T+C) x \ni 0$ follows from the solvability of $(T+C) x+\epsilon x \ni 0$ because the latter says that $0 \in \overline{R(T+C)}$, but we have assumed that $(T+C) \bar{G}$ is a closed set.

Condition (a) in Theorem 4 can be used in Theorem 5 to ensure that $R(T+C)$ is a closed set. We cannot use condition (b) of Theorem 4 because the compactness of the resolvent $J_{1}$ implies that the set $\left[J_{1}(T+I)\right] G_{0}=G_{0}$ is relatively compact, i.e., the space $X$ is finite-dimensional. The set $G_{0}$ here is a sufficiently small open set such that $(T+I) G_{0}$ is bounded. It is a well-known fact that a demicontinuous accretive mapping is locally bounded whenever the space $X^{*}$ is assumed to be uniformly convex (cf. [23, Section 3]).

Open Problem 1. Is Theorem 5 true without the assumption that $[(I+T)(\partial G)] \cap$ $\operatorname{int} G=\emptyset$ ?

\section{Perturbations of Maximal Monotone Operators}

In this section we study perturbation problems

$$
T x+C x \ni 0,
$$

where $T: X \supset D(T) \rightarrow 2^{X^{*}}$ is now a maximal monotone operator and $C: X \supset$ $D(T) \rightarrow X^{*}$ is at least bounded. As it is usually assumed in this setting, the space $X$ is reflexive and the spaces $X, X^{*}$ are locally uniformly convex. Thus, $J$ is now a bicontinuous mapping. If $X, X^{*}$ are uniformly convex, then $J: X \rightarrow$ $X^{*}, J^{-1}: X^{*} \rightarrow X^{* *}$ are uniformly continuous on bounded sets. An operator $T: X \supset D(T) \rightarrow 2^{X^{*}}$ is "monotone" if for every $x, y \in D(T)$ and $u^{*} \in T x, v^{*} \in T y$ we have

$$
\left\langle u^{*}-v^{*}, x-y\right\rangle \geq 0 .
$$

A monotone operator is "maximal monotone" if $T+\lambda J$ is surjective for all $\lambda>0$. An operator $T: X \supset D(T) \rightarrow 2^{X^{*}}$ is said to be of "type $(S)$ " on the set $E \subset D(T)$, if for every sequence $\left\{x_{n}\right\} \subset E$ with $x_{n} \rightarrow x_{0} \in X$ and

$$
\lim _{n \rightarrow \infty}\left\langle v_{n}^{*}, x_{n}-x_{0}\right\rangle=0
$$

for some $v_{n}^{*} \in T x_{n}$, we have $x_{n} \rightarrow x_{0}$. It is said to be of "type $\left(S_{+}\right)$" on the set $E \subset D(T)$ if for every sequence $\left\{x_{n}\right\} \subset E$ with $x_{n} \rightarrow x_{0} \in X$ and

$$
\limsup _{n \rightarrow \infty}\left\langle v_{n}^{*}, x_{n}-x_{0}\right\rangle \leq 0
$$

for some $v_{n}^{*} \in T x_{n}$, we have $x_{n} \rightarrow x_{0}$. It is well-known that, under our assumptions on the spaces $X, X^{*}$, the duality mapping $J$ is of type $\left(S_{+}\right)$on $X$.

For fundamental properties of monotone operators, and other related concepts, the reader is referred to Barbu [2], Browder [5], Cioranescu [7], Pascali and Sburlan [35] and Zeidler [41]. We start this section with an extension of Theorem 2. 
Theorem 6. Let $T: X \supset D(T) \rightarrow 2^{X^{*}}$ be maximal monotone and (i) $C: D(T) \rightarrow$ $X^{*}$ compact. Assume that there exist $\beta \in \Gamma$ and $Q>0$ such that: for every $x \in$ $D(T)$ with $\|x\| \geq Q$ and every $v^{*} \in T x$ we have

$$
\left\langle v^{*}+C x, x\right\rangle \geq-\beta(\|x\|)\|x\|^{2} .
$$

Then $R(T+C+\epsilon J)=X^{*}$, for every $\epsilon \in(0,1)$. Assume, further, that

$$
\liminf _{\substack{\|x\| \rightarrow \infty \\ x \in D(T)}} \frac{|T x+C x|}{\|x\|}>0 .
$$

Then $\overline{R(T+C)}=X^{*}$. Let one of the following conditions hold:

(a) $C: \overline{D(T)} \rightarrow X^{*}$ is completely continuous;

(b) instead of (i), $C: \overline{D(T)} \rightarrow X^{*}$ is bounded and continuous, and $(T+J)^{-1}$ is compact.

Then $R(T+C)=X^{*}$.

Proof. As in the proof of Theorem 2, we consider the homotopy inclusion

$$
t(T x+C x+\epsilon J) x+(1-t) J x \ni t s,
$$

where $t \in[0,1], \epsilon \in(0,1)$ and $s \in X^{*}$. This inclusion can be written as

$$
u^{*}=H\left(t, u^{*}\right) \equiv-t\left[C(t(T+\epsilon J)+(1-t) J)^{-1} u^{*}-s\right],
$$

where

$$
x=(t(T+\epsilon J)+(1-t) J)^{-1} u^{*} .
$$

We remark here that $T_{t} \equiv t(T+\epsilon J)+(1-t) J: D(T) \rightarrow 2^{X^{*}}$ is a surjective maximal monotone operator with inverse $T_{t}^{-1}$ which is defined, bounded and continuous on all of $X^{*}$. The maximal monotonicity/surjectivity of $T_{t}$ can be seen from

$$
T_{t} \equiv t T+[1-t(1-\epsilon)] J,
$$

because $1-t(1-\epsilon) \geq \epsilon, t \in[0,1]$. The continuity of the inverse $T_{t}^{-1}$ follows from Cioranescu [7, Proposition 3.10]. To see its boundedness, fix $t \in(0,1]$. If $t=0$, the boundedness of $T_{t}^{-1}$ is obvious. Fix $x_{0} \in D(T), v_{0}^{*} \in T x_{0}$, and let $\left\{y_{n}^{*}\right\} \subset X^{*}$ be a bounded sequence. Let

$$
x_{n}=(t(T+\epsilon J)+(1-t) J)^{-1} y_{n}^{*} .
$$

Then, for some $v_{n}^{*} \in T x_{n}$,

$$
t v_{n}^{*}+[1-t(1-\epsilon)] J x_{n}=y_{n}^{*},
$$

or

$$
[t / \lambda(t)] v_{n}^{*}+J x_{n}=[1 / \lambda(t)] y_{n}^{*},
$$

where $\lambda(t) \equiv 1-t(1-\epsilon), t \in[0,1]$. Now, we evaluate (24) at the point $x_{n}-x_{0}$ to get

$$
[t / \lambda(t)]\left\langle v_{n}^{*}, x_{n}-x_{0}\right\rangle+\left\langle J x_{n}^{*}, x_{n}-x_{0}\right\rangle=[1 / \lambda(t)]\left\langle y_{n}^{*}, x_{n}-x_{0}\right\rangle,
$$


Or

$$
\begin{aligned}
{[t / \lambda(t)]\left\langle v_{n}^{*}-v_{0}^{*}, x_{n}-x_{0}\right\rangle } & +[t / \lambda(t)]\left\langle v_{0}^{*}, x_{n}-x_{0}\right\rangle \\
& +\left\langle J x_{n}-J x_{0}, x_{n}-x_{0}\right\rangle+\left\langle J x_{0}, x_{n}-x_{0}\right\rangle \\
& =[1 / \lambda(t)]\left\langle y_{n}^{*}, x_{n}-x_{0}\right\rangle .
\end{aligned}
$$

Using the monotonicity of $T$ and the fact that

$$
\left\langle J x_{n}-J x_{0}, x_{n}-x_{0}\right\rangle \geq\left(\left\|x_{n}\right\|-\left\|x_{0}\right\|\right)^{2},
$$

we obtain

$$
\left(\left\|x_{n}\right\|-\left\|x_{0}\right\|\right)^{2} \leq\left[[t / \lambda(t)]\left\|v_{0}^{*}\right\|+\left\|J x_{0}\right\|+[1 / \lambda(t)]\left\|y_{n}^{*}\right\|\right]\left\|x_{n}-x_{0}\right\|,
$$

which shows the boundedness of the sequence $\left\{x_{n}\right\}$. Hence, $T_{t}^{-1}$ is bounded.

We can further rewrite (23) as follows:

$$
\begin{aligned}
u^{*}=H\left(t, u^{*}\right) & =-t\left[C(t T+(1-t(1-\epsilon)) J)^{-1} u^{*}-s\right] \\
& =-t\left[C([t /(1-t(1-\epsilon))] T+J)^{-1}[1 /(1-t(1-\epsilon))] u^{*}-s\right] \\
& =-t\left[C((t / \lambda(t)) T+J)^{-1}\left[(1 / \lambda(t)) u^{*}\right]-s\right]
\end{aligned}
$$

where $H\left(0, u^{*}\right) \equiv 0$. The function $H(t, \cdot)$ is compact, for every $t \in[0,1]$, under any one of the assumptions made on the operators $C,(T+J)^{-1}$. In fact, it was shown in [17] (see, also, the resolvent identity in the comments following the proof of Theorem 8 below) that the compactness of the resolvent $(T+J)^{-1}$ implies the compactness of every resolvent $(k T+J)^{-1}, k>0$. We are going to show, later in this proof, that the set

$$
S \equiv \overline{H\left([0,1], \overline{B_{r}(0)}\right)}
$$

is compact, for every $r>0$.

In order to show the continuity of $H\left(t, u^{*}\right)$ on any set $[0,1] \times \overline{B_{r}(0)}, r>0$, we set $Q \equiv B_{q}(0) \subset X^{*}$ and observe that the set

$$
K=\bigcup_{t \in[0,1]}\left\{((t / \lambda(t)) T+J)^{-1} \bar{Q}\right\}
$$

is bounded. In fact, let $\left\{x_{n}\right\} \subset K$. Then there exist $\left\{t_{n}\right\} \subset[0,1]$ and $\left\{u_{n}^{*}\right\} \subset \bar{Q}$ such that

$$
x_{n}=\left(\left(t_{n} / \lambda\left(t_{n}\right)\right) T+J\right)^{-1} u_{n}^{*} .
$$

We have, for $q(t) \equiv t / \lambda(t)$ and some $v_{n}^{*} \in T x_{n}$,

$$
q\left(t_{n}\right) v_{n}^{*}+J x_{n}=u_{n}^{*}
$$

This implies

$$
\begin{aligned}
q\left(t_{n}\right)\left\langle v_{n}^{*}-v_{0}^{*}, x_{n}-x_{0}\right\rangle+ & q\left(t_{n}\right)\left\langle v_{0}^{*}, x_{n}-x_{0}\right\rangle \\
& +\left\langle J x_{n}-J x_{0}, x_{n}-x_{0}\right\rangle+\left\langle J x_{0}, x_{n}-x_{0}\right\rangle \\
= & \left\langle u_{n}^{*}, x_{n}-x_{0}\right\rangle
\end{aligned}
$$


where $x_{0} \in D(T)$ and $v_{0}^{*} \in T x_{0}$. This inequality implies

$$
\left(\left\|x_{n}\right\|-\left\|x_{0}\right\|\right)^{2} \leq\left[q\left(t_{n}\right)\left\|v_{0}^{*}\right\|+\left\|J x_{0}\right\|+\left\|u_{n}^{*}\right\|\right]\left\|x_{n}-x_{0}\right\|,
$$

and the boundedness of $\left\{x_{n}\right\}$, with a bound depending only on $\left\|v_{0}^{*}\right\|,\left\|x_{0}\right\|$ and $q$, follows from the boundedness of $\left\{u_{n}^{*}\right\}$.

From the boundedness of the set $K$ we have immediately that the set

$$
L \equiv \bigcup_{t \in[0,1]}\left\{(q(t) T+J)^{-1}\left[[1 / \lambda(t)] \overline{B_{r}(0)}\right]\right\}
$$

is bounded, for each $r>0$, for there exists a ball $B_{q}(0)$ such that

$$
\bigcup_{t \in[0,1]}\left\{[1 / \lambda(t)] B_{r}(0)\right\} \subset B_{q}(0), t \in[0,1] .
$$

Consequently, by the boundedness of $C$, the set

$$
M \equiv \bigcup_{t \in[0,1]}\left\{C(q(t) T+J)^{-1}\left[[1 / \lambda(t)] \overline{B_{r}(0)}\right]\right\}=C L
$$

is bounded. Letting $\mu(r)$ be an upper bound for the set $M$, we have

$$
\left\|H\left(t, u^{*}\right)\right\| \leq t[\mu(r)+\|s\|], t \in[0,1],
$$

which shows the continuity of the function $H\left(\cdot, u^{*}\right)$ at $t=0$, uniformly w.r.t. $u^{*} \in \overline{B_{r}(0)}$. It also shows the continuity of $H\left(t, u^{*}\right)$ at any point $\left(0, u^{*}\right), u^{*} \in \overline{B_{r}(0)}$.

To see the continuity of $H\left(t, u^{*}\right)$ at any point $\left(t_{0}, u_{0}^{*}\right) \in(0,1] \times \overline{B_{r}(0)}$, we fix such a point and let $\left(t_{n}, u_{n}^{*}\right) \in[0,1] \times \overline{B_{r}(0)}$ be such that $t_{n} \rightarrow t_{0}$ and $u_{n}^{*} \rightarrow u_{0}^{*}$ as $n \rightarrow \infty$. Naturally, we may assume that $t_{n}>0, n=1,2, \ldots$. Let

$$
\begin{aligned}
& v_{n}^{*} \equiv H\left(t_{n}, u_{n}^{*}\right)=t_{n}\left[C\left(q\left(t_{n}\right) T+J\right)^{-1}\left(p\left(t_{n}\right) u_{n}^{*}\right)-s\right], \\
& v_{0}^{*} \equiv H\left(t_{0}, u_{0}^{*}\right)=t_{0}\left[C\left(q\left(t_{0}\right) T+J\right)^{-1}\left(p\left(t_{0}\right) u_{0}^{*}\right)-s\right],
\end{aligned}
$$

where $p(t)=1 / \lambda(t), t \in(0,1]$. Let

$$
\begin{aligned}
x_{n} & \equiv\left(q\left(t_{n}\right) T+J\right)^{-1}\left(p\left(t_{n}\right) u_{n}^{*}\right) \\
x_{0} & \equiv\left(q\left(t_{0}\right) T+J\right)^{-1}\left(p\left(t_{0}\right) u_{0}^{*}\right) .
\end{aligned}
$$

We have, for some $z_{n}^{*} \in T x_{n}, z_{0}^{*} \in T x_{0}$,

$$
\begin{aligned}
q\left(t_{n}\right) z_{n}^{*}+J x_{n} & =p\left(t_{n}\right) u_{n}^{*}, \\
q\left(t_{0}\right) z_{0}^{*}+J x_{0} & =p\left(t_{0}\right) u_{0}^{*},
\end{aligned}
$$

which leads to

$$
\begin{aligned}
\left\langle q\left(t_{n}\right) z_{n}^{*}-q\left(t_{n}\right) z_{0}^{*}, x_{n}-x_{0}\right\rangle & +\left[q\left(t_{n}\right)-q\left(t_{0}\right)\right]\left\langle z_{0}^{*}, x_{n}-x_{0}\right\rangle \\
& +\left\langle J x_{n}-J x_{0}, x_{n}-x_{0}\right\rangle \\
& =\left\langle p\left(t_{n}\right) u_{n}^{*}-p\left(t_{0}\right) u_{0}^{*}, x_{n}-x_{0}\right\rangle .
\end{aligned}
$$


We note that $\left\{x_{n}\right\}$ is a bounded sequence because the sequence $\left\{p\left(t_{n}\right) u_{n}^{*}\right\}$ is bounded and the sequence $\left\{x_{n}\right\}$ lies in the set $L$ above, which is also bounded. Letting $\nu$ denote an upper bound for $\left\{x_{n}\right\}$ and using (27), we obtain

It follows that

$$
\begin{aligned}
\left\langle J x_{n}-J x_{0}, x_{n}-x_{0}\right\rangle \leq & \left|q\left(t_{n}\right)-q\left(t_{0}\right)\right|\left\|z_{0}^{*}\right\|\left\|x_{n}-x_{0}\right\| \\
& +\left\|p\left(t_{n}\right) u_{n}^{*}-p\left(t_{0}\right) u_{0}^{*}\right\|\left\|x_{n}-x_{0}\right\| \\
\leq & {\left[\left|q\left(t_{n}\right)-q\left(t_{0}\right)\right|\left\|z_{0}^{*}\right\|+\left\|p\left(t_{n}\right) u_{n}^{*}-p\left(t_{0}\right) u_{0}^{*}\right\|\right]\left(\nu+\left\|x_{0}\right\|\right) . }
\end{aligned}
$$

$$
\limsup _{n \rightarrow \infty}\left\langle J x_{n}-J x_{0}, x_{n}-x_{0}\right\rangle \leq 0 .
$$

Since $J$ is of type $\left(S_{+}\right)$, we have $x_{n} \rightarrow x_{0}$. Using this property in

$$
\begin{aligned}
\left\|v_{n}^{*}-v_{0}^{*}\right\| & \leq\left\|t_{n} C x_{n}-t_{0} C x_{0}\right\|+\left|t_{n}-t_{0}\right|\|s\| \\
& \leq\left|t_{n}-t_{0}\right|\left\|C x_{n}\right\|+t_{0}\left\|C x_{n}-C x_{0}\right\|+\left|t_{n}-t_{0}\right|\|s\|,
\end{aligned}
$$

along with the boundedness and the continuity of $C$, we obtain the desired continuity property of $H\left(t, u^{*}\right)$.

We now show the compactness of the set $S$. If the operator $C: D(T) \rightarrow X^{*}$ is compact, then the boundedness of the set $L$ implies that the set $C L$ is relatively compact. This implies that the set $C L-s$ is also relatively compact. Letting $U \equiv$ $[0,1] \cdot \overline{C L-s}$, we see that $U$ is a compact set because it is the image of the set $[0,1] \times \overline{C L-s}$ by the operation of scalar multiplication. Since $S \subset U$, we have the compactness of the set $S$. If, on the other hand, $C: \overline{D(T)} \rightarrow X^{*}$ is continuous and bounded and the resolvent $(T+J)^{-1}$ is compact, then the compactness of the set $S$ follows exactly as in the corresponding part of the proof of Theorem 2. It is therefore omitted. The degree function $d\left(I-H(t, \cdot), B_{r}(0), 0\right)$ is well-defined and the rest of the proof follows the corresponding proof steps of Theorem 2 . We only make a comment for the case (a). In case (a) we use the fact that $\overline{D(T)}$ is a convex set. Thus, whenever $\left\{x_{n}\right\} \subset D(T)$ with $x_{n} \rightarrow x_{0} \in \overline{D(T)}$, then $C x_{n} \rightarrow C x_{0}$. Moreover, $v_{n}^{*} \rightarrow-C x_{0}+s \in T x_{0}$, where $v_{n}^{*} \in T x_{n}$ satisfies

$$
v_{n}^{*}+C x_{n}+(1 / n) J x_{n}=s .
$$

In order to be able to use homotopies as in Theorems 3 and 4 , but for maximal monotone operators, we need the Yosida approximants for maximal monotone mappings introduced by Brézis, Crandall and Pazy [4] (cf. also Barbu [2, p. 41] and Pascali and Sburlan [35, p. 128]). Given a maximal monotone operator $T: X \supset D(T) \rightarrow 2^{X^{*}}, x \in X$ and $\lambda>0$, there exists a unique $x_{\lambda} \in D(T)$ such that

$$
J\left(x_{\lambda}-x\right)+\lambda T x_{\lambda} \ni 0 .
$$

We set

$$
J_{\lambda} x=x_{\lambda} \text { and } T_{\lambda} x=\frac{1}{\lambda} J\left(x-x_{\lambda}\right) .
$$

We know that $J_{\lambda}: X \rightarrow X, T_{\lambda}: X \rightarrow X^{*}$ are single-valued and bounded operators. We also know that both operators are demicontinuous with $T_{\lambda}$ maximal monotone. In addition, it is easy to see that $T_{\lambda} x \in T J_{\lambda} x, x \in X$. The demicontinuity of $J_{\lambda}$ can be found in the proof of Proposition 1.1 of Barbu [2]. The rest of these properties can also be found in [2], [4] and [35].

Since it is generally easier to check the compactness of $(\lambda T+J)^{-1}$ than that of $J_{\lambda}$, we establish the following lemma. 
Lemma 3. For a maximal monotone operator $T: X \supset D(T) \rightarrow 2^{X^{*}}$, the resolvent $J_{\lambda}$ is compact if and only if $(\lambda T+J)^{-1}$ is compact. Consequently, if $J_{\lambda}$ is compact for some $\lambda>0$, the same is true for all $\lambda \in(0, \infty)$.

Proof. Given $x \in X$ and $\lambda>0$ such that (29) holds, we have

$$
x \in x_{\lambda}+\lambda J^{-1} T x_{\lambda} .
$$

This gives us the following representation for $J_{\lambda}$ :

$$
J_{\lambda} x=\left(I+\lambda J^{-1} T\right)^{-1} x, x \in X .
$$

We show first that if $(\lambda T+J)^{-1}$ is compact, then so is $J_{\lambda}$. To this end, let $(\lambda T+J)^{-1}$ be compact, for some $\lambda>0$, let $\left\{x_{n}\right\}$ be a bounded sequence in $X$, and let

$$
y_{n} \equiv J_{\lambda} x_{n}=\left(I+\lambda J^{-1} T\right)^{-1} x_{n} .
$$

Then

$$
\lambda J^{-1} T y_{n}+y_{n} \ni x_{n},
$$

or

$$
\lambda T y_{n}+J y_{n} \ni J y_{n}+J\left(x_{n}-y_{n}\right) .
$$

This produces the representation

$$
y_{n}=(\lambda T+J)^{-1}\left(J y_{n}+J\left(x_{n}-y_{n}\right)\right) .
$$

Since the sequences $\left\{x_{n}\right\},\left\{y_{n}\right\}$ and $J$ are bounded and $(\lambda T+J)^{-1}$ is compact, we have that $\left\{y_{n}\right\}$ lies in a compact set. Thus, $J_{\lambda}$ maps bounded sets into relatively compact sets. We now show that $J_{\lambda}$ is also continuous independently of the fact that it maps bounded sets onto relatively compact sets. Let $\left\{x_{n}\right\} \subset X$ be such that $x_{n} \rightarrow x_{0}$. Then

$$
y_{n} \equiv J_{\lambda} x_{n} \rightarrow y_{0} \equiv J_{\lambda} x_{0} .
$$

We have

$$
J\left(y_{n}-x_{n}\right)+\lambda T y_{n} \ni 0, J\left(y_{0}-x_{0}\right)+\lambda T y_{0} \ni 0 .
$$

Thus, for some $v_{n}^{*} \in T y_{n}, v_{0}^{*} \in T y_{0}$,

$$
\begin{aligned}
\left\langle J\left(y_{n}-x_{n}\right)-\right. & \left.J\left(y_{0}-x_{0}\right),\left(y_{n}-x_{n}\right)-\left(y_{0}-x_{0}\right)\right\rangle \\
= & \left\langle J\left(y_{n}-x_{n}\right)-J\left(y_{0}-x_{0}\right),\left(y_{n}-y_{0}\right)-\left(x_{n}-x_{0}\right)\right\rangle \\
= & \left\langle J\left(y_{n}-x_{n}\right)-J\left(y_{0}-x_{0}\right), y_{n}-y_{0}\right\rangle \\
& -\left\langle J\left(y_{n}-x_{n}\right)-J\left(y_{0}-x_{0}\right), x_{n}-x_{0}\right\rangle \\
\leq & -\lambda\left\langle v_{n}^{*}-v_{0}^{*}, y_{n}-y_{0}\right\rangle+\left\|J\left(y_{n}-x_{n}\right)-J\left(y_{0}-x_{0}\right)\right\|\left\|x_{n}-x_{0}\right\| \\
\leq & \left\|J\left(y_{n}-x_{n}\right)-J\left(y_{0}-x_{0}\right)\right\|\left\|x_{n}-x_{0}\right\| .
\end{aligned}
$$

This says that

$$
\limsup _{n \rightarrow \infty}\left\langle J\left(y_{n}-x_{n}\right)-J\left(y_{0}-x_{0}\right),\left(y_{n}-x_{n}\right)-\left(y_{0}-x_{0}\right)\right\rangle \leq 0 .
$$


Since

$$
\lim _{n \rightarrow \infty}\left\langle J\left(y_{0}-x_{0}\right),\left(y_{n}-x_{n}\right)-\left(y_{0}-x_{0}\right)\right\rangle=0,
$$

because $y_{n}-x_{n} \rightarrow y_{0}-x_{0}$, we obtain

$$
\limsup _{n \rightarrow \infty}\left\langle J\left(y_{n}-x_{n}\right),\left(y_{n}-x_{n}\right)-\left(y_{0}-x_{0}\right)\right\rangle \leq 0 .
$$

Since $J$ is of type $\left(S_{+}\right)$, we have $y_{n}-x_{n} \rightarrow y_{0}-x_{0}$, i.e., $y_{n} \rightarrow y_{0}$.

Conversely, let $J_{\lambda}$ be compact, for some $\lambda>0$. We already know that the operator $(\lambda T+J)^{-1}$ is continuous. To show its compactness, let $\left\{x_{n}^{*}\right\} \subset X^{*}$ be bounded and let

$$
x_{n}=(\lambda T+J)^{-1} x_{n}^{*} .
$$

Then

$$
\lambda T x_{n}+J x_{n} \ni x_{n}^{*}
$$

or

$$
x_{n}+\lambda J^{-1} T x_{n} \ni x_{n}+J^{-1}\left(x_{n}^{*}-J x_{n}\right),
$$

which produces the representation

$$
x_{n}=\left(I+\lambda J^{-1} T\right)^{-1}\left(x_{n}+J^{-1}\left(x_{n}^{*}-J x_{n}\right)\right) .
$$

It follows that since $\left\{x_{n}\right\},\left\{x_{n}^{*}\right\}, J$ and $J^{-1}$ are bounded and $\left(I+\lambda J^{-1} T\right)^{-1}$ is compact, we have that $\left\{x_{n}\right\}$ lies in a compact set. Thus, $(\lambda T+J)^{-1}$ is compact.

Guan and the author have shown in [15] that the compactness of $(\lambda T+J)^{-1}$, for some $\lambda>0$, implies the compactness of $(\mu T+J)^{-1}$ for every $\mu>0$. In view of the above equivalence, we have that if $J_{\lambda}$ is compact, for some $\lambda>0$, then $J_{\mu}$ is compact for all $\mu>0$.

Using the resolvents $J_{\lambda}$, we can now establish Theorem 7 below, which extends to the maximal monotone case the main result of the author in [28]. All the results in that reference are for $m$-accretive operators.

Theorem 7. Let $T: X \supset D(T) \rightarrow 2^{X^{*}}$ be maximal monotone and $C: D(T) \rightarrow$ $X^{*}$. Let $(T+J)^{-1}$ be compact. Let $G \subset X$ be open, bounded and such that, for some $z \in D(T) \cap G$ and some $v^{*} \in T z$,

$$
T x-v^{*} \not \supset 0 \text { and }\left\langle u^{*}+C x, x-z\right\rangle>0, \quad\left(x, u^{*}\right) \in(D(T) \cap \partial G) \times T x .
$$

Assume, further, that the operator $C(\lambda T+J)^{-1}$ is compact, where $\lambda$ is a fixed positive constant, and the set $C(D(T) \cap \bar{G})$ is bounded. Then $0 \in(T+C)(D(T) \cap \bar{G})$.

Proof. We are planning to solve the perturbed problem

$$
T J_{\lambda} x+C J_{\lambda} x \ni 0 .
$$

Using the fact that $T_{\lambda} x \in T J_{\lambda} x$, it suffices to solve instead of (32) the equation

$$
T_{\lambda} x+C J_{\lambda} x=0 .
$$


Using the definition of $T_{\lambda}$ from (30), we see that (33) is equivalent to

$$
x=J_{\lambda} x-\lambda J^{-1} C J_{\lambda} x .
$$

We note that the operator $-\lambda J^{-1} C J_{\lambda}$ is compact because it is the composition of the compact mapping $C J_{\lambda}$ with the homeomorphism $-\lambda J^{-1}$. The compactness of $C J_{\lambda}$ follows from the compactness of $C(\lambda T+J)^{-1}$ and the fact that

$$
C J_{\lambda} x=C(\lambda T+J)^{-1}\left[J J_{\lambda} x+J\left(x-J_{\lambda} x\right)\right],
$$

which follows from the representation of $y_{n}$ in the proof of Lemma 3 . Since $J_{\lambda}$ is compact by Lemma 3, the right-hand side of (34) defines a compact mapping.

We show first that we may assume that $z=0 \in D(T) \cap G$ and $0 \in T(0)$. In fact, if this is not true, we consider the new operators $\widetilde{T}, \widetilde{C}$ defined by

$$
\widetilde{T} x \equiv T(x+z)-v^{*}, \quad \widetilde{C} x \equiv C(x+z)+v^{*}, \quad x \in \widetilde{D}(T),
$$

where $v^{*}$ is as in the statement of the theorem and $D(\widetilde{T}) \equiv D(T)-z$. We also set $\widetilde{G} \equiv G-z$. It is easy to see that the operator $\widetilde{T}$ is maximal monotone on $\widetilde{D}(T)$. To show the compactness of the resolvent

$$
\widetilde{S}_{1} \equiv(J+\widetilde{T})^{-1},
$$

and hence any resolvent $(J+\lambda \widetilde{T})^{-1}$, we note first that $\widetilde{S}_{1}$ is a continuous mapping, by the maximal monotonicity of the operator $\widetilde{T}$. Let $\left\{y_{n}^{*}\right\}$ be a bounded sequence in $X^{*}$ and let $x_{n}=\widetilde{S}_{1} y_{n}^{*}$. Then

$$
y_{n}^{*}=J x_{n}+\widetilde{v}_{n}^{*}=J x_{n}+v_{n}^{*}-v^{*}, \quad n=1,2, \ldots,
$$

where $\widetilde{v}_{n}^{*} \in \widetilde{T} x_{n}$ and $v_{n}^{*} \in T\left(x_{n}+z\right)$. Thus,

$$
J\left(x_{n}+z\right)+T\left(x_{n}+z\right) \ni y_{n}^{*}+J\left(x_{n}+z\right)-J x_{n}+v^{*},
$$

or

$$
x_{n}=(J+T)^{-1}\left[y_{n}^{*}+J\left(x_{n}+z\right)-J x_{n}+v^{*}\right]-z .
$$

We can now invoke the boundedness of the sequences $\left\{x_{n}\right\},\left\{y_{n}^{*}\right\}$ and the duality mapping $J$, as well as the compactness of the operator $(J+T)^{-1}$, in order to concluded that $\left\{x_{n}\right\}$ lies in a compact set. This proves the compactness of the operator $\widetilde{S}_{1}$.

We now show that the operator $-\lambda J^{-1} \widetilde{C}\left(I+\lambda J^{-1} \widetilde{T}\right)^{-1}$ is compact. To this end, we seek to express this operator in terms of the operator $C(I+\lambda T)^{-1}$. In fact, letting

$$
\widetilde{J}_{\lambda} \equiv\left(I+\lambda J^{-1} \widetilde{T}\right)^{-1}
$$

and

$$
x \equiv \widetilde{J}_{\lambda} y=\left(I+\lambda J^{-1} \widetilde{T}\right)^{-1} y \in D(\widetilde{T}),
$$

for some $y \in X$, we have that there exists $w \in D(T)$ such that $x=w-z$ and

$$
y \in x+\lambda \widetilde{J}^{-1} \widetilde{T} x=x+\lambda J^{-1}\left(T(x+z)-v^{*}\right)=w-z+\lambda J^{-1}\left[T(w)-v^{*}\right] .
$$


Thus,

$$
J(y+z-w) \in \lambda\left(T w-v^{*}\right)
$$

and

$$
J^{-1}\left(J(y+z-w)+\lambda v^{*}\right) \in \lambda J^{-1} T w .
$$

Consequently,

$$
w=\left(I+\lambda J^{-1} T\right)^{-1}\left(w+J^{-1}\left(J(y+z-w)+\lambda v^{*}\right)\right) .
$$

Letting $x=w-z$ and using the above representation of $w$, we obtain

$$
\begin{aligned}
x & =\widetilde{J}_{\lambda} y=\left(I+\lambda J^{-1} T\right)^{-1}\left[x+z+J^{-1}\left(J(y-x)+\lambda v^{*}\right)\right]-z \\
& =J_{\lambda}\left[\widetilde{J}_{\lambda} y+z+J^{-1}\left[J\left(I-\widetilde{J}_{\lambda}\right) y+\lambda v^{*}\right]\right]-z \\
& =J_{\lambda} Q y-z,
\end{aligned}
$$

where $Q y$ is the expression in the brackets to the right of the first $J_{\lambda}$ in (35). Since

$$
\widetilde{C} \widetilde{J}_{\lambda} y=C\left(\widetilde{J}_{\lambda} y+z\right)+v^{*}=C J_{\lambda} Q y+v^{*}
$$

and $Q$ is continuous and bounded, we obtain that $-\lambda J^{-1} \widetilde{C} \widetilde{J}_{\lambda}$ is still a compact operator. To see that the second condition in (31) is satisfied with $z=0$, it suffices to observe that

$$
\left\langle\left(w^{*}-v^{*}\right)+\left(C(u+z)+v^{*}\right), u\right\rangle \geq 0,
$$

for every $u \in D(\widetilde{T}) \cap \partial \widetilde{G}$ and every $w^{*} \in T(u+z)$. We note that the first condition (31) becomes now

$$
0 \notin \widetilde{T} x, x \in D(\widetilde{T}) \cap \partial \widetilde{G} .
$$

Finally, to show that the set $\widetilde{C}(D(\widetilde{T}) \cap \overline{\widetilde{G}})$ is bounded, we note that

$$
\begin{aligned}
\widetilde{C}(D(\widetilde{T}) \cap \overline{\widetilde{G}}) & =\widetilde{C}[(D(T)-z) \cap(\overline{G-z})] \\
& =C[((D(T)-z) \cap(\bar{G}-z))+z]+v^{*} \\
& =C(D(T) \cap \bar{G})+v^{*} .
\end{aligned}
$$

We have shown that it suffices to prove the theorem with $z=0$ and $0 \in T(0)$.

Now, we let

$$
U=\left(I+\lambda J^{-1} T\right)(D(T) \cap G)
$$

and consider the homotopy functions

$$
H_{1}(t, x) \equiv x-J_{\lambda} x+t \lambda J^{-1} C J_{\lambda} x, \quad(t, x) \in[0,1] \times \bar{U},
$$

and

$$
H_{2}(t, x) \equiv x-t J_{\lambda} x, \quad(t, x) \in[0,1] \times \bar{U} .
$$

Since the operator $J_{\lambda}$ is a continuous mapping on all of $X$, its inverse, $I+\lambda J^{-1} T$, is a set-valued mapping that maps relatively open (closed) sets of its domain $D(T)$ 
onto open (closed) sets of the space $X$. Because of this, the set $U$ is open and the set

$$
\left(I+\lambda J^{-1} T\right)(D(T) \cap \bar{G})
$$

is closed. Thus, we have

$$
\begin{aligned}
& \overline{\left(I+\lambda J^{-1} T\right)(D(T) \cap \bar{G})} \\
& =\left(I+\lambda J^{-1} T\right)(D(T) \cap \bar{G}) \\
& =\left(I+\lambda J^{-1} T\right)(D(T) \cap G) \cup\left(I+\lambda J^{-1} T\right)(D(T) \cap \partial G) \\
& \supset \overline{\left(I+\lambda J^{-1} T\right)(D(T) \cap G)} \\
& =\left(I+\lambda J^{-1} T\right)(D(T) \cap G) \cup \partial\left(\left(I+\lambda J^{-1} T\right)(D(T) \cap G)\right),
\end{aligned}
$$

which implies that

$$
\left(I+\lambda J^{-1} T\right)(D(T) \cap \partial G) \supset \partial\left(\left(I+\lambda J^{-1} T\right)(D(T) \cap G)\right) .
$$

Since

$$
\overline{\left(I+\lambda J^{-1} T\right)(D(T) \cap G)} \subset\left(I+\lambda J^{-1} T\right)(D(T) \cap \bar{G}),
$$

the homotopies $H_{1}(t, y), H_{2}(t, y)$ are well-defined. They also are compact displacements of the identity in $y$. Since $J_{\lambda}$ maps the set $\bar{U}$ onto a bounded subset of $D(T) \cap \bar{G}$ and $C(D(T) \cap \bar{G})$ is relatively compact, the operators $J_{\lambda}-t \lambda J^{-1} C J_{\lambda}$ and $t J_{\lambda}$ have relatively compact range on $\bar{U}$. We may thus apply the Leray-Schauder degree function $D_{L S}$ (cf. Nagumo [34]) to the mappings $H_{1}(t, \cdot), H_{2}(t, \cdot)$. We are going to show first that

$$
d_{L S}\left(H_{1}(1, \cdot), U, 0\right)=d_{L S}\left(H_{2}(1, \cdot), U, 0\right),
$$

and then prove that

$$
d_{L S}\left(H_{2}(1, \cdot), U, 0\right)=d_{L S}(I, U, 0)=1,
$$

the last equality holding because $0 \in U$. As we shall see below, to show our first assertion, it suffices to prove that the equation $H_{1}(t, x)=0$ has no solution $x$ on $\partial U$. Assume that the contrary is true. Then there exists $x_{t} \in \partial U$ such that

$$
x_{t}=J_{\lambda} x_{t}-t \lambda J^{-1} C J_{\lambda} x_{t} .
$$

We may assume that $t \in[0,1)$. In fact, if $t=1$, then we have a solution $x_{1}$ of (34) which provides a solution to our problem (32). Equality (38) can also be written as

$$
T_{\lambda} x_{t}+t C J_{\lambda} x_{t}=0 .
$$

This says that if $y_{t} \equiv J_{\lambda} x_{t}$, then there exists $w_{t}^{*} \in T y_{t}$ such that

$$
w_{t}^{*}+t C y_{t}=0 .
$$

Since $y_{t} \in D(T) \cap \partial G$ and $T x \not \supset 0$ for $x \in D(T) \cap \partial G$, we have that $t \in(0,1)$. Hence,

$$
\left\langle w_{t}^{*}+t C y_{t}, y_{t}\right\rangle=\left\langle w_{t}^{*}, y_{t}\right\rangle+t\left\langle C y_{t}, y_{t}\right\rangle=0
$$


We consider the straight line segment $l(u)=a u+b, u \in[0,1]$, where $a \equiv\left\langle C y_{t}, y_{t}\right\rangle$ and $b \equiv\left\langle w_{t}^{*}, y_{t}\right\rangle$. We know that $l(0) \geq 0$, because $0 \in T(0)$ and $T$ is monotone. We also know that $l(1)>0$, because of our assumed boundary condition. It follows that $l(u)>0$, for every $u \in(0,1)$. This contradicts (39). Hence

$$
d_{L S}\left(H_{1}(1, \cdot), U, 0\right)=d_{L S}\left(H_{1}(0, \cdot), U, 0\right)=d_{L S}\left(H_{2}(1, \cdot), U, 0\right) .
$$

In order to prove (37), we are going to show that the equation $H_{2}(t, x)=0$ has no solution $x \in \partial U$. Let $x_{t}$ be such a solution for some $t \in[0,1]$. Obviously, $t \neq 0$ because $x_{t} \in \partial U$, while $0 \in U$ and $U$ is an open set. Also, $t \neq 1$. In fact, if $t=1$, then $x_{t}-J_{\lambda} x_{t}=0$ implies

$$
0=\frac{1}{\lambda} J\left(x_{t}-J_{\lambda} x_{t}\right)=T_{\lambda} x_{t} \in T\left(J_{\lambda} x_{t}\right) \subset T(D(T) \cap \partial G),
$$

i.e., a contradiction to our assumption. Thus, $t \in(0,1)$ and

$$
x_{t}=t J_{\lambda} x_{t} .
$$

Letting $y_{t} \equiv J_{\lambda} x_{t}$, we see that

$$
y_{t}+\lambda J^{-1} T y_{t} \ni t y_{t} .
$$

This implies

$$
\lambda T y_{t} \ni-(1-t) J y_{t},
$$

which yields, for some $w_{t} \in T y_{t}$,

$$
0 \leq \lambda\left\langle w_{t}^{*}, y_{t}\right\rangle=-(1-t)\left\langle J y_{t}, y_{t}\right\rangle=-(1-t)\left\|y_{t}\right\|^{2}<0,
$$

the last inequality holding because $t \in(0,1)$ and $y_{t} \in D(T) \cap \partial G$. It follows that (37) is true. This says that $H_{1}(1, x)$ is solvable in $x \in U$. The proof is complete.

If $z=0$ in Theorem 7 , we obtain the following simpler corollary.

Corollary 4. Let $T: X \supset D(T) \rightarrow 2^{X^{*}}$ be maximal monotone, with $0 \in D(T)$ and $0 \in T(0)$, and $C: D(T) \rightarrow X^{*}$. Let $(T+J)^{-1}$ be compact. Let $G \subset X$ be open, bounded and such that $0 \in G$ and

$$
T x \not \ngtr 0 \text { and }\left\langle u^{*}+C x, x\right\rangle>0, \quad\left(x, u^{*}\right) \in(D(T) \cap \partial G) \times T x .
$$

Assume, further, that the operator $C(\lambda T+J)^{-1}$ is compact, where $\lambda$ is a fixed positive constant, and the set $C(D(T) \cap \bar{G})$ is bounded. Then $0 \in(T+C)(D(T) \cap \bar{G})$.

For condensing mappings $C J_{\lambda}$, we have the following version of Theorem 7 .

Theorem 8. Let $T: X \supset D(T) \rightarrow 2^{X^{*}}$ be maximal monotone, with $0 \in D(T)$ and $0 \in T(0)$. Assume that the duality mapping $J^{-1}$ is Lipschitzian with Lipschitz constant $K>0$, and let $J_{1}$ be compact. Let $G \subset X$ be open, bounded and such that $0 \in G$ and

$$
T x \not \ngtr 0 \text { and }\left\langle u^{*}+C x, x\right\rangle>0, \quad\left(x, u^{*}\right) \in(D(T) \cap \partial G) \times T x .
$$


Assume that the operator $C\left(I+\lambda J^{-1} T\right)^{-1}$ is condensing, where $\lambda$ is a fixed constant in $(0,1 / K)$, and the set $C(D(T) \cap \bar{G})$ is bounded. Then $0 \in(T+C)(D(T) \cap \bar{G})$.

Proof. The proof follows as in Theorem 7. However, the operator $-\lambda J^{-1} C J_{\lambda}$ is now condensing, while the operator $J_{\lambda}$ is still compact. Since

$$
J_{\lambda} \bar{U} \subset D(T) \cap \bar{G}
$$

and

$$
\left(C J_{\lambda}\right)(\bar{U}) \subset C(D(T) \cap \bar{G}),
$$

we have that the operators $J_{\lambda}$ and $-\lambda J^{-1} C J_{\lambda}$ have bounded ranges on $\bar{U}$. As a consequence of this fact, we are allowed to use a degree theory for the mappings $H_{1}(t, x), H_{2}(t, x)$ which can be found in Theorem 1 of [28]. That theorem contains a sketch of the proof of the existence of such a degree theory.

It is of importance to note that the operator $C$ in Theorems 7 and 8 is not assumed to be continuous. This is why we are precluded from using homotopies like the ones used by the author in [22-24], the author and Liu [29] and Guan and the author [15-17]. The study of operators $C$ which are not assumed to satisfy any continuity property was initiated recently by Hirano and Kalinde [19]. For an application of this setting, we refer to that paper as well as the paper of the author $[28]$.

The compactness of the operator $C J_{\lambda}$, in Theorem 7 and Corollary 4, implies the compactness of every operator $C J_{\mu}, \mu>0$. To see this, we write $J_{\mu}$ in terms of $J_{\lambda}$ in the following way. Let $x=J_{\mu} y$, for some $y \in X$. Then we have $x+\mu J^{-1} T x \ni y$ and

$$
(1 / \mu) x+J^{-1} T x \ni(1 / \mu) y .
$$

Hence

$$
x+(\lambda / \mu) x+\lambda J^{-1} T x \ni x+(\lambda / \mu) y,
$$

or

$$
x=J_{\mu} y=J_{\lambda}\left(\frac{\lambda}{\mu} y+\frac{\mu-\lambda}{\mu} x\right)=J_{\lambda}\left(\frac{\lambda}{\mu} y+\frac{\mu-\lambda}{\mu} J_{\mu} y\right) .
$$

This is a "resolvent identity" for the mappings $J_{\lambda}$. Since $J_{\mu}$ is a compact mapping, this identity shows that if $C J_{\lambda}$ is compact, then so is $C J_{\mu}$, for every $\mu>0$.

Due to the above comments, we could have used $J_{1}$ in Theorem 7 , and its proof, instead of $J_{\lambda}$. However, we need the proof of Theorem 7, as it stands, for the proof of Theorem 8 which imitates it.

Calvert and Gupta have shown in [6, Lemma 1.1] that the normalized duality mapping $J: L^{p}(\Omega) \rightarrow L^{q}(\Omega)$ satisfies a Lipschitz condition, provided that $p \in$ $[2, \infty)$. Thus, Theorem 8 applies to the space $X=L^{q}(\Omega)$. Here, $\Omega$ is a bounded domain in $\mathcal{R}^{n}$ and $(1 / p)+(1 / q)=1$.

Open Problem 2. Is Theorem 7 true without the assumption that $T x-v^{*} \not \supset 0$, for every $x \in D(T) \cap \partial G$ ?

We are now going to study the effect of another boundary condition on the range of the sum of two operators. This condition implies condition (22) of Theorem 6 and is accompanied by a growth condition on the sum $T+C$ which is weaker than $(* * *)$. Because of these facts, we have the immediate solvability of $(T+C+\epsilon J) x \ni p$, for all $\epsilon \in(0,1)$, but we do not have the surjectivity of the operator $T+C+\epsilon J$ as a consequence of Theorem 6 . 
Theorem 9. Let $T: X \supset D(T) \rightarrow 2^{X^{*}}$ be maximal monotone and (a) $C: D(T) \rightarrow$ $X^{*}$ compact. Let $S \subset X^{*}$. Assume that for every $s \in S$ there exist three positive constants $a=a(s), q=q(s), Q=Q(s)$ such that

$$
a>1+\frac{\|s\|}{q}
$$

and the following two conditions are satisfied:

(i) $\left\|u^{*}+C x\right\| \geq a q$, for every $x \in D(T)$ with $\|x\| \geq Q$, $u^{*} \in T x$;

(ii) we have

$$
\left\langle u^{*}+C x-s, x\right\rangle \geq-q\|x\|, \text { for every } x \in D(T) \text { with }\|x\| \geq Q
$$

and every $u^{*} \in T x$.

Then $S \subset \overline{R(T+C)}$. If, moreover, $C: \overline{D(T)} \rightarrow X^{*}$ is completely continuous, then $S \subset R(T+C)$. This is also true if instead of (a) it is assumed that $C: \overline{D(T)} \rightarrow X^{*}$ is continuous and bounded, and $(J+T)^{-1}$ is compact.

Proof. We consider the approximate problem

$$
T x+C x+[(q+\epsilon) / Q] J x \ni s,
$$

where $s$ is a given point in the set $S$ and $\epsilon \in(0,1)$ is chosen so that

$$
a>1+\frac{\|s\|+\epsilon}{q} .
$$

We note that in our assumptions we may use any number greater than $Q$, instead of $Q$ itself. Thus, we may assume that $Q$ is large enough so that $Q>q+\epsilon$. Consequently, the solvability of the problem (40), for any $s \in X^{*}$, follows from Theorem 6 because, by (ii),

$$
\left\langle u^{*}+C x, x\right\rangle \geq-[(\|s\|+q) /\|x\|]\|x\|^{2}=-\beta(\|x\|)\|x\|^{2},
$$

for every $x \in D(T)$ with $\|x\| \geq Q, u^{*} \in T x$, where

$$
\beta(\rho) \equiv(\|s\|+q) / \rho \rightarrow 0 \text { as } \rho \rightarrow \infty .
$$

To show that every solution $x$ of (40) has to lie in the ball $B_{Q}(0)$, assume that the contrary is true, and let $x$ solve (40) with $\|x\| \geq Q$. Then, for some $u^{*} \in T x$,

$$
\begin{aligned}
0 & =\left\langle u^{*}+C x-s, x\right\rangle+[(q+\epsilon) / Q]\langle J x, x\rangle \\
& \geq-q\|x\|+[(q+\epsilon) / Q]\|x\|^{2} \\
& \geq-q\|x\|+[(q+\epsilon) / Q] Q\|x\| \\
& =\epsilon\|x\| \\
& >0
\end{aligned}
$$

i.e., a contradiction. Let $\left\{Q_{n}\right\}$ be a sequence of numbers in $(0, \infty)$ such that $Q_{n} \geq Q, Q_{n} \rightarrow \infty$ as $n \rightarrow \infty$, and there exists a solution $x_{n}$ of the problem

$$
T x+C x+\left[(q+\epsilon) / Q_{n}\right] J x \ni s
$$


with $x_{n} \in B_{Q_{n}}(0)$. This is possible by what was shown above. Let us assume that $\left\|x_{n}\right\| \geq Q$, for some $n$. Then, for some $u_{n}^{*} \in T x_{n}$, we have

$$
\begin{aligned}
0 & =\left\|u_{n}^{*}+C x_{n}+\left[(q+\epsilon) / Q_{n}\right] J x_{n}-s\right\| \\
& \geq\left\|u_{n}^{*}+C x_{n}\right\|-\left[\left[(q+\epsilon) / Q_{n}\right]\left\|J x_{n}\right\|+\|s\|\right] \\
& >a q-\left[\left[(q+\epsilon) / Q_{n}\right] Q_{n}+\|s\|\right] \\
& =a q-(q+\epsilon+\|s\|) \\
& =\left[a-\left(1+\frac{\|s\|+\epsilon}{q}\right)\right] q \\
& >0
\end{aligned}
$$

i.e., a contradiction. Since $\left\{J x_{n}\right\}$ is a bounded sequence, we have immediately that $s \in \overline{R(T+C)}$ and $S \subset \overline{R(T+C)}$. The rest of the proof follows exactly as in Theorem 6 . It is therefore omitted.

A special case of the assumptions of Theorem 9 allows for a generally better conclusion. Namely, in the proof of Theorem $9, s \in \overline{(T+C)\left(D(T) \cap B_{Q(s)}(0)\right)}$. This case is contained in the following result.

Theorem 9'. Let $T: X \supset D(T) \rightarrow 2^{X^{*}}$ be maximal monotone with $0 \in D(T)$ and (a) $C: D(T) \rightarrow X^{*}$ compact. Let $S \subset X^{*}$. Assume that for every $s \in S$ there exist three positive constants $a=a(s), q=q(s), Q=Q(s)$ such that

$$
a>1+\frac{\|s\|}{q}
$$

and the following two conditions are satisfied:

(i) $\left\|u^{*}+C x\right\| \geq a q$, for every $x \in D(T)$ with $\|x\| \geq Q, u^{*} \in T x$;

(ii) we have

$$
\left\langle C x+v^{*}-s, x\right\rangle \geq-q\|x\|, \text { for every } x \in D(T) \text { with }\|x\| \geq Q
$$

and every $u^{*} \in T x$.

Then, for every $s \in S, s \in \overline{(T+C)\left(D(T) \cap B_{Q(s)}(0)\right)}$. If, moreover, $C$ is completely continuous, then, for every $s \in S, s \in(T+C)\left(D(T) \cap \overline{B_{Q(s)}(0)}\right)$. This is also true if instead of (a) it is assumed that $C$ is continuous and bounded, and $(J+T)^{-1}$ is compact.

Proof. Unlike the proof of Theorem 9, we no longer need to impose a lower bound on the parameter $Q$. In fact, we now use the homotopy equation

$$
u^{*}=H\left(t, u^{*}\right) \equiv-t\left[\widetilde{C}(\widetilde{T}+(q / Q) J)^{-1} u^{*}-s\right]
$$

where $\widetilde{T} x \equiv T x-v^{*}$ and $\widetilde{C} x \equiv C x+v^{*}$. The rest of the proof follows as in Theorem 14 of [29]. It is therefore omitted. -

We are now ready for the following maximal monotone analogue of Theorem 14 in $[29]$. 
Corollary 5. Let $T: X \supset D(T) \rightarrow 2^{X^{*}}$ be maximal monotone and (a) $C: D(T) \rightarrow$ $X^{*}$ compact. Assume that $0 \in D(T)$ and there exist three positive constants $a_{1}, b, r$ and $v^{*} \in T(0)$ such that

$$
a_{1}>2+\frac{r}{\left\|v^{*}\right\|+r}
$$

and the following conditions are satisfied.

(i) $\left\|u^{*}+C x\right\| \geq a_{1}\left(\left\|v^{*}\right\|+r\right)$, for every $x \in D(T)$ with $\|x\| \geq b$, $u^{*} \in T x$;

(ii) $\langle C x, x\rangle \geq-\left(\left\|v^{*}\right\|+r\right)\|x\|$, for every $x \in D(T)$ with $\|x\| \geq b$.

Then $\overline{B_{r}(0)} \subset \overline{(T+C)\left(D(T) \cap B_{b}(0)\right)}$. If, moreover, $C: \overline{D(T)} \rightarrow X^{*}$ is completely continuous, then $\overline{B_{r}(0)} \subset(T+C)\left(D(T) \cap \overline{B_{b}(0)}\right)$. This is also true if instead of (a) it is assumed that $C: \overline{D(T)} \rightarrow X^{*}$ is continuous and bounded, and $(J+T)^{-1}$ is compact.

Proof. In Theorem $9^{\prime}$, we take $S=\overline{B_{r}(0)}, q=2\left(\left\|v^{*}\right\|+r\right), Q=b$, and $a=a_{1} / 2$. We see here that the parameters $q, Q$ and $a$ do not depend on the particular point $s \in S$. For every $u^{*} \in T x$ with $\|x\| \geq Q$ and every $s \in S$ we have

$$
\begin{aligned}
\left\langle u^{*}+C x-s, x\right\rangle & =\left\langle u^{*}-v^{*}+C x+v^{*}-s, x\right\rangle \\
& =\left\langle u^{*}-v^{*}, x\right\rangle+\langle C x, x\rangle+\left\langle v^{*}-s, x\right\rangle \\
& \geq-\left(\left\|v^{*}\right\|+r\right)\|x\|-\left(\left\|v^{*}\right\|+\|s\|\right)\|x\| \\
& \geq-2\left(\left\|v^{*}\right\|+r\right)\|x\| .
\end{aligned}
$$

Thus,

$$
a=a_{1} / 2>1+\frac{r}{2\left(\left\|v^{*}\right\|+r\right)} \geq 1+\frac{\|s\|}{q} .
$$

Finally, we have a new surjectivity result for perturbations of maximal monotone operators. For a set $A$, we set $|A| \equiv \inf \{\|x\|: x \in A\}$.

Corollary 6. Let $T: X \supset D(T) \rightarrow 2^{X^{*}}$ be maximal monotone with $0 \in D(T)$ and (a) $C: D(T) \rightarrow X^{*}$ compact. Assume that the following two conditions hold:

(i)

$$
\lim _{\substack{\|x\| \rightarrow \infty \\ x \in D(T)}}|T x+C x|=\infty
$$

(ii) there exists a constant $d>0$ such that $\langle C x, x\rangle \geq-d\|x\|$, for every $x \in D(T)$ with sufficiently large norm.

Then $\overline{R(T+C)}=X^{*}$. If, moreover, $C: \overline{D(T)} \rightarrow X^{*}$ is completely continuous, then $R(T+C)=X^{*}$. This is also true if instead of (a) it is assumed that $C: \overline{D(T)} \rightarrow X^{*}$ is continuous and bounded, $(J+T)^{-1}$ is compact.

Proof. Given a ball $S \equiv \overline{B_{r}(0)} \subset X^{*}$, for some $r>0$, we let $s \in S, v^{*} \in T(0)$ and $u^{*} \in T x$, where $x \in D(T)$ has a sufficiently large norm. We have

$$
\begin{aligned}
\left\langle u^{*}-v^{*}+C x+v^{*}-s, x\right\rangle & \geq-\left(d+\left\|v^{*}\right\|+\|s\|\right)\|x\| \\
& \geq-\left(d+\left\|v^{*}\right\|+r\right)\|x\| \\
& =-q\|x\|,
\end{aligned}
$$

where $q$ is an obvious positive constant. Since $|T x+C x| \geq a q$, for all large numbers $a>0$ and all large $\|x\|$, we obtain that $\overline{B_{r}(0)} \subset \overline{R(T+C)}$. Since $r>0$ is arbitrary, we have that $\overline{R(T+C)}=X^{*}$. The rest of the proof follows as before. It is therefore omitted. 


\section{Eigenvalues for Perturbations of Maximal Monotone Operators}

The Yosida resolvents $J_{\lambda}=\left(I+\lambda J^{-1} T\right)^{-1}$ may also be used in the solvability of nonlinear eigenvalue problems involving perturbations of maximal monotone operators. Since such resolvents were not used in [16], we find it instructive to establish some results in this direction. Again, $X$ is a real reflexive Banach space and $X, X^{*}$ are locally uniformly convex. We also assume that the space $X$ is infinite dimensional. We do this because of Lemma A below, which can be found in [16].

Lemma A. Let $K \subset X^{*}$ be compact and assume that $\left\|x^{*}\right\| \geq \alpha, x^{*} \in K$, where $\alpha$ is a positive constant. Then there exists $y_{0}^{*} \in \partial B_{1}(0)$ such that $\left\{t y_{0}^{*}: t \geq \alpha\right\} \cap K=\emptyset$.

The next result is an interesting extension of the maximal monotone analogue of Theorem 2.5 in [16].

Theorem 10. Let $G \subset X$ be open, bounded, and let $T: X \supset D(T) \rightarrow 2^{X^{*}}$ be maximal monotone and such that $0 \in D(T) \cap G, 0 \in T(0)$ and $0 \notin T\left(J_{\mu}(\partial G)\right)$, for some $\mu>0$. Assume that $(J+T)^{-1}$ is compact. Let $C: D(T) \rightarrow X^{*}$ be such that $C J_{1}$ is compact and $\left\|C J_{\mu} x\right\| \geq \alpha, x \in \partial G$, where $\alpha$ is a positive constant. Then there exists $\left(\lambda_{0}, x_{0}\right) \in(0, \infty) \times D(T)$ such that $C x_{0} \neq 0$ and $T x_{0}-\lambda_{0} C x_{0} \ni 0$.

Proof. We consider first the problem

$$
T_{\mu} x-\lambda C J_{\mu} x=0, \quad(\lambda, x) \in(0, \infty) \times \partial G .
$$

This problem can be written as

$$
H(\lambda, x) \equiv x-J_{\mu} x-\lambda \mu J^{-1} C J_{\mu} x=0, \quad(\lambda, x) \in(0, \infty) \times \partial G .
$$

We know that $J_{\mu}$ is compact, by Lemma 3 , and that the operator $C J_{\mu}$ is also compact by the comments preceding the statement of Theorem 9 . In order to solve (42) in $(\lambda, x) \in(0, \infty) \times \partial G$, we show first that there exists $\lambda_{0} \in(0, \infty)$ such that

$$
x-J_{\mu} x-\mu J^{-1}\left(\lambda_{0} C J_{\mu} x+\eta y_{0}^{*}\right) \neq 0,(\eta, x) \in(0, \infty) \times \partial G,
$$

where $y_{0}^{*}$ is as in Lemma A with $K \equiv \overline{-\left(C J_{\mu}\right)(\partial G)}$. The operator

$$
x \rightarrow J_{\mu} x+\mu J^{-1}\left(\lambda_{0} C J_{\mu} x+\eta y_{0}^{*}\right)
$$

is compact for every $\eta \in[0, \infty)$. The set $K$ is compact because $\partial G$ is bounded and the operator $C J_{\mu}$ is compact.

Let us assume that there is no $\lambda_{0}>0$ such that (43) holds. Then there exist sequences $\left\{\lambda_{m}\right\} \subset(0, \infty),\left\{\eta_{m}\right\} \subset(0, \infty)$ and $\left\{x_{m}\right\} \subset \partial G$ such that $\lambda_{m} \rightarrow \infty$ and

$$
x_{m}-J_{\mu} x_{m}-\mu J^{-1}\left(\lambda_{m} C J_{\mu} x_{m}+\eta_{m} y_{0}^{*}\right)=0, \quad m=1,2, \ldots .
$$

Thus,

$$
\frac{1}{\mu} J\left(x_{m}-J_{\mu} x_{m}\right)-\left(\lambda_{m} C J_{\mu} x_{m}+\eta_{m} y_{0}^{*}\right)=0, \quad m=1,2, \ldots,
$$

or

$$
T_{\mu} x_{m}-\left(\lambda_{m} C J_{\mu} x_{m}+\eta_{m} y_{0}^{*}\right)=0 .
$$


Since $\left\{T_{\mu} x_{m}\right\}$ is a bounded sequence, we obtain

$$
\lim _{m \rightarrow \infty}\left[C J_{\mu} x_{m}+\left(\eta_{m} / \lambda_{m}\right) y_{0}^{*}\right]=0
$$

Since $\left\|C J_{\mu} x_{m}\right\| \geq \alpha$ for all $m$ and $\left\{C J_{\mu} x_{m}\right\}$ is bounded, we may assume that $\left\{\eta_{m} / \lambda_{m}\right\}$ converges to some number $\nu \geq 0$. Since $C J_{\mu}$ is compact, we may also assume that $C J_{\mu} x_{m} \rightarrow u^{*}$ with $u^{*}=-\nu y_{0}^{*}$. Since

$$
\alpha \leq\left\|u^{*}\right\|=\nu\left\|y_{0}^{*}\right\|=\nu
$$

and $\nu y_{0}^{*} \in \overline{-\left(C J_{\mu}\right)(\partial G)}$, we have a contradiction to the choice of $y_{0}^{*}$. Consequently, (43) is true. If (42) holds for $\lambda=\lambda_{0}$ and some $x \in \partial G$, then we have the solvability of the problem (41). Otherwise, (43) holds for all $\eta \geq 0$. Assuming that the latter is the case, we show that the Leray-Schauder degree $d\left(H\left(\lambda_{0}, \cdot\right), G, 0\right)=0$. To this end, let $d\left(H\left(\lambda_{0}, \cdot\right), G, 0\right) \neq 0$ and consider the homotopy

$$
H_{1, m}(t, x) \equiv x-J_{\mu} x-\mu J^{-1}\left(\lambda_{0} C J_{\mu} x+t m y_{0}^{*}\right), t \in[0,1], x \in \bar{G}, m=1,2, \ldots .
$$

We know that $H_{1, m}(t, \partial G) \not \supset 0, t \in[0,1]$. It follows that the Leray-Schauder degree $d\left(H_{1, m}(t, \cdot), G, 0\right)$ is well-defined, and we have

$$
d\left(H_{1, m}(1, \cdot), G, 0\right)=d\left(H_{1, m}(0, \cdot), G, 0\right)=d\left(H\left(\lambda_{0}, \cdot\right), G, 0\right) \neq 0 .
$$

This implies that there exists a sequence $\left\{x_{m}\right\} \subset G$ such that $H_{1, m}\left(1, x_{m}\right)=0$ or

$$
T_{\mu} x_{m}-\lambda_{0} C J_{\mu} x_{m}=m y_{0}^{*} .
$$

This is a contradiction to the boundedness of the sequence

$$
\left\{\left(T_{\mu}-\lambda_{0} C J_{\mu}\right) x_{m}\right\} .
$$

It follows that $d\left(H\left(\lambda_{0}, \cdot\right), G, 0\right)=0$.

We now show that $d(H(0, \cdot), G, 0)=1$. Consider the new homotopy

$$
H_{2}(t, x) \equiv x-t J_{\mu} x,(t, x) \in[0,1] \times \bar{G} .
$$

Assume that $x_{t}$ is a solution of $H_{2}(t, x)=0$ with $x_{t} \in \partial G$. Let

$$
u_{t} \equiv J_{\mu} x_{t}=\left(I+\mu J^{-1} T\right)^{-1} x_{t} \in D(T) .
$$

Then there exists $v_{t}^{*} \in T u_{t}$ such that

$$
u_{t}+\mu J^{-1} v_{t}^{*}=x_{t}
$$

and

Since $0 \in T(0)$, we have

$$
v_{t}^{*}=-\frac{1}{\mu}(1-t) J u_{t} .
$$

$$
0 \leq\left\langle v_{t}^{*}, u_{t}\right\rangle=-\frac{1}{\mu}(1-t)\left\langle J u_{t}, u_{t}\right\rangle=-\frac{1}{\mu}(1-t)\left\|u_{t}\right\|^{2} \leq 0 .
$$

Thus, either $t=1$ or $u_{t}=0$. In either case, $0=v_{t}^{*} \in T u_{t}=T J_{\mu} x_{t}$. Since $x_{t} \in \partial G$, we have a contradiction. It follows that the equation $H_{2}(t, x)=0$ has no solutions $x \in \partial G$, for any value of $t \in[0,1]$. Thus, the degree $d_{2}(H(t, \cdot), G, 0)$ is welldefined, constant and equal to 1 because $d\left(H_{2}(t, \cdot), G, 0\right)=d\left(H_{2}(0, \cdot), G, 0\right)=1$. Consequently, there exists $\left(\lambda_{0}, x\right) \in(0, \infty) \times \partial G$ such that $H\left(\lambda_{0}, x\right)=0$, or

$$
T_{\mu} x-\lambda_{0} C J_{\mu} x=0 .
$$

Letting $x_{0}=J_{\mu} x$, we have $\left\|C x_{0}\right\| \geq \alpha>0$ and $T x_{0}-\lambda_{0} C x_{0} \ni 0$. This completes the proof.

We give Corollary 7 below because we want to comment on the boundary condition imposed upon the operator $C$ in a special case. 
Corollary 7. Let the assumptions of Theorem 10 be satisfied, but with the operator $C: \overline{D(T)} \rightarrow X^{*}$ and the compactness of the operator $C J_{1}$ replaced by " $C$ is continuous." Then there exists $\left(\lambda_{0}, x_{0}\right) \in(0, \infty) \times D(T)$ such that $C x_{0} \neq 0$ and $T x_{0}-\lambda_{0} C x_{0} \ni 0$.

The condition $\left\|C J_{\mu} x\right\| \geq \alpha, x \in \partial G$, will hold in Corollary 7 if we assume instead that $C x \neq 0, x \in \overline{D(T)}$. In fact, let us assume that the latter is true and that

$$
\inf _{x \in \partial G}\left\{\left\|C J_{\mu} x\right\|\right\}=0 .
$$

Then there exists a sequence $\left\{x_{m}\right\} \subset \partial G$ such that $C J_{\mu} x_{m} \rightarrow 0$ as $m \rightarrow \infty$. Since $J_{\mu}$ is compact, we may (and do) assume that $J_{\mu} x_{m} \rightarrow u_{0} \in \overline{D(T)}$. Since $C$ : $\overline{D(T)} \rightarrow X^{*}$ is continuous, $C J_{\mu} x_{m} \rightarrow C u_{0}=0$, i.e., a contradiction.

If

$$
L \equiv \sup _{x \in \partial G}\{\|x\|\}
$$

it suffices to assume, in the appropriate places in Theorem 10 and Corollary 7 , that $0 \notin T\left(D(T) \cap \overline{B_{2 L}(0)}\right)$ and $\|C x\| \geq \alpha, x \in D(T) \cap \overline{B_{2 L}(0)}$. In fact, on page 43 of Barbu's book [2] we find

$$
\left\|J_{\mu} x-x\right\|^{2} \leq\|x-u\|\left\|J_{\mu} x-x\right\|+\mu\|x-u\|\|v\|+\mu\|v\|\left\|x-J_{\mu} x\right\|,
$$

for any $u \in D(T)$ and $v \in T u$. Since $0 \in T(0)$, we may take $u=0, v=0$ to obtain $\left\|J_{\mu} x-x\right\| \leq\|x\|$, which says that $\left\|J_{\mu} x\right\| \leq 2\|x\| \leq 2 L, x \in \partial G$. Consequently, $J_{\mu}(\partial G) \subset \overline{B_{2 L}(0)}$. In particular, if $G=B_{r}(0)$, then

$$
T\left(J_{\mu}(\partial G)\right) \subset T\left(D(T) \cap \overline{B_{2 r}(0)}\right) \quad \text { and } \quad C\left(J_{\mu}(\partial G)\right) \subset C\left(D(T) \cap \overline{B_{2 r}(0)}\right) .
$$

This assumption on the operator $C$ requires it to be bounded below away from zero only on the ball $\overline{B_{2 L}(0)}$, and is considerably weaker than the one above involving the entire set $\overline{D(T)}$. This is particularly important when the operator $T$ is densely defined.

If the set $T(D(T) \cap \bar{G})$ is bounded, then we can have an eigenvalue result under the more natural assumption $\|C x\| \geq \alpha, x \in D(T) \cap \partial G$.

Theorem 11. Let $G \subset X$ be open and bounded. Let $T: X \supset D(T) \rightarrow 2^{X^{*}}$ be maximal monotone with $0 \in D(T) \cap G, 0 \in T(0)$ and $0 \notin T(D(T) \cap \partial G)$. Assume that the set $T(D(T) \cap \bar{G})$ is bounded. Assume, further, that $C: D(T) \rightarrow X^{*}$ is such that $C J_{1}$ is compact, $(J+T)^{-1}$ is compact, and there exists $\alpha>0$ such that $\|C x\| \geq$ $\alpha, x \in D(T) \cap \partial G$. Then for every $\mu>0$ there exists $\left(\lambda_{0}, x_{0}\right) \in(0, \infty) \times(D(T) \cap \partial G)$ such that $T x_{0}-\lambda_{0} C x_{0} \ni 0$.

Proof. We follow, generally, the proof of Theorem 10. The mapping $J_{\mu} \equiv$ $\left(I+\mu J^{-1} T\right)^{-1}: X \rightarrow D(T)$ is continuous. This was shown in the proof of Lemma 3. Thus, its inverse image, $I+\mu J^{-1} T$, maps relatively open subsets of its domain $D(T)$ onto open sets in $X$. Consequently, the set $\left(I+\mu J^{-1} T\right)(D(T) \cap G)$ is open and the set $\left(I+\mu J^{-1} T\right)(D(T) \cap \bar{G})$ is closed in $X$. Let

$$
U \equiv\left(I+\mu J^{-1} T\right)(D(T) \cap G) .
$$


Then, as before,

$$
\partial U \subset\left(I+\mu J^{-1} T\right)(D(T) \cap \partial G)
$$

and

$$
\bar{U} \subset\left(I+\mu J^{-1} T\right)(D(T) \cap \bar{G}) .
$$

In order to solve (42) with $x \in \partial U$, we observe first that the operator

$$
C J_{\mu}: \bar{U} \rightarrow X^{*}
$$

is compact. We also observe that if $x \in \partial U$, then

$$
J_{\mu} x \in J_{\mu}\left(I+\mu J^{-1} T\right)(D(T) \cap \partial G)=D(T) \cap \partial G .
$$

This equality is implied by the existence of the single-valued inverse $J_{\mu}$. Thus,

$$
\left\|C J_{\mu} x\right\| \geq \alpha .
$$

We set

$$
K \equiv \overline{-C J_{\mu} \partial U}
$$

We let $\left\{\lambda_{m}\right\},\left\{\eta_{m}\right\},\left\{x_{m}\right\}$ be as in the proof of Theorem 10, but with

$$
\left\{x_{m}\right\} \subset \partial U \subset\left(I+\mu J^{-1} T\right)(D(T) \cap \partial G) .
$$

Since $J^{-1}$ is a bounded mapping and the set $D(T) \cap \bar{G}$ is bounded, we have that the set $\left(I+\mu J^{-1} T\right)(D(T) \cap \partial G)$ is bounded. This says that $\left\{x_{m}\right\}$ is a bounded sequence. Moreover, $\left\{T_{\mu} x_{m}\right\}$ is bounded because of the boundedness of the operator $T_{\mu}$. Continuing as in Theorem 10, we obtain that $d\left(H_{2}(0, \cdot), U, 0\right)=d(I, U, 0)=1$, which implies that there exists

$\left(\lambda_{0}, x\right) \in(0, \infty) \times \partial\left(\left(I+\mu J^{-1} T\right)(D(T) \cap G)\right) \subset(0, \infty) \times\left(\left(I+\mu J^{-1} T\right)(D(T) \cap \partial G)\right)$

such that

$$
T_{\mu} x-\lambda_{0} C J_{\mu} x=0 .
$$

Letting $x_{0}=J_{\mu} x$, we have our conclusion.

Open Problem 3. Is Theorem 10 true with $\|C x\| \geq \alpha, x \in \partial G$, instead of $\left\|C J_{\mu} x\right\| \geq \alpha, x \in \partial G$ ?

\section{More General Approximating Inclusions in Hilbert Spaces}

A basic fact in the theory of maximal monotone and $m$-accretive operators is that the approximating problems that are usually employed are of the "first power" with respect to $x$. By this, we mean that we add either $\epsilon J x$, in the monotone case, or $\epsilon x$, in the accretive case, to the sum of the operators involved in order to solve an approximating problem which is easier to handle than the original one. One of the reasons for doing this is that we want to exploit the properties of resolvents $(\lambda T+I)^{-1}$, in the accretive case, and $(\lambda T+J)^{-1}$ in the monotone case. It is therefore natural to ask whether other "small" approximating terms could actually produce more general results than the ones heretofore obtained. We call the attention of the 
reader to the fact that we are not discussing here the introduction of $K$-monotone mappings, which aims, in part, at a unified theory of accretive and monotone operators. We are actually referring to the fact that most of the "inner-product" and "norm" criteria established until now are tied to the fact that the small terms of the respective stabilizing problems are of the first power with respect to $x$. Attempts with other small perturbing terms were made by various authors in their efforts to show that the solutions of the stabilizing (or approximating) problems converge to the solution of the original problem. A good number of such references are listed in the paper of Alber [1]. Alber himself considered the problem of approximating a solution of an equation of the type

$$
T x=f,
$$

where $T: X \rightarrow X^{*}$ is maximal monotone and $f \in X^{*}$, by a solution $x_{\epsilon}$ of the stabilizing problem

$$
T x+\epsilon U x=f .
$$

The interesting feature of that paper is that the operator $U$ is given by $U x \equiv$ $\operatorname{grad}\|x\|^{\gamma}$, for some $\gamma>1$. Alber showed that $x_{\epsilon} \rightarrow x_{0}$, where $x_{\epsilon}$ solves the above equation, $T x_{0}=f$, and

$$
\left\|x_{0}\right\|=\inf \{\|x\|: T x=f\} .
$$

It is our intention here to introduce the mapping

$$
\widetilde{J} x \equiv\|x\|^{p} x, \quad x \in H,
$$

in the perturbation theory of nonlinear operators in real Hilbert spaces $H$. Here, $p$ is a fixed positive constant. This mapping is a continuous, maximal monotone operator satisfying the coercivity condition

$$
\langle\widetilde{J} x-\widetilde{J} y, x-y\rangle \geq \frac{1}{2^{p}}\|x-y\|^{p+2}, \quad x, y \in H .
$$

Actually, the constant $1 / 2^{p}$ is best possible (cf. [30]). Consequently, the mapping $\widetilde{J}$ is a homeomorphism. Its inverse is given, for $y \neq 0$, by

$$
\widetilde{J}^{-1} y=\frac{y}{\|y\|^{p /(p+1)}}, \quad y \in H,
$$

with

$$
\left\|\widetilde{J}^{-1} y\right\|=\|y\|^{1 /(p+1)} .
$$

Using this mapping $\widetilde{J}$, we can define the Yosida approximants, $\widetilde{J}_{\lambda}, \widetilde{T}_{\lambda}$, exactly as before, but with $\widetilde{J}$ in place of $J$. Thus, we have

$$
\widetilde{J}\left(\widetilde{J}_{\lambda} x-x\right)+\lambda \widetilde{T}_{\lambda} x=0, \quad x \in X .
$$

Again, $\widetilde{J}_{\lambda}: X \rightarrow D(T), \widetilde{T}_{\lambda}: X \rightarrow X^{*}$, and it is easy to see that

$$
\widetilde{J}_{\lambda} x=\left(I+\lambda^{1 /(p+1)} \widetilde{J}^{-1} T\right)^{-1}, \quad \widetilde{T}_{\lambda} x=(1 / \lambda) \widetilde{J}\left(x-\widetilde{J}_{\lambda} x\right) .
$$


Both mappings $\widetilde{J}_{\lambda}, \widetilde{T}_{\lambda}$ are continuous and bounded. Moreover, the mapping $\widetilde{J}$ is odd and positively homogeneous of degree $p+1$. In order to obtain a resolvent identity for the resolvents $\widetilde{J}_{\lambda}$, we observe that

$$
\begin{aligned}
\widetilde{J}_{\lambda}^{-1} \widetilde{J}_{\mu} x & =\left(I+\lambda^{1 /(p+1)} \widetilde{J}^{-1} T\right) \widetilde{J}_{\mu} x \\
& =\widetilde{J}_{\mu} x+\lambda^{1 /(p+1)} \widetilde{J}^{-1} T \widetilde{J}_{\mu} x \\
& \supset \widetilde{J}_{\mu} x+\lambda^{1 /(p+1)} \widetilde{J}^{-1} \widetilde{T}_{\mu} x \\
& =\widetilde{J}_{\mu} x+\lambda^{1 /(p+1)} \widetilde{J}^{-1}\left[\frac{1}{\mu} \widetilde{J}\left(x-\widetilde{J}_{\mu} x\right)\right] \\
& =\widetilde{J}_{\mu} x+\frac{\lambda^{1 /(p+1)}}{\mu^{1 /(p+1)}} \widetilde{J}^{-1} \widetilde{J}\left(x-\widetilde{J}_{\mu} x\right), \\
& =\widetilde{J}_{\mu} x+\frac{\lambda^{1 /(p+1)}}{\mu^{1 /(p+1)}}\left(x-\widetilde{J}_{\mu} x\right),
\end{aligned}
$$

i.e.,

$$
\widetilde{J}_{\mu} x=\widetilde{J}_{\lambda}\left[\frac{\lambda^{1 /(p+1)}}{\mu^{1 /(p+1)}} x+\frac{\mu^{1 /(p+1)}-\lambda^{1 /(p+1)}}{\mu^{1 /(p+1)}} \widetilde{J}_{\mu} x\right] .
$$

This is the desired resolvent identity. It implies, among other things, the compactness of $\widetilde{J}_{\mu}$, for all $\mu>0$, if we know the compactness of the resolvent for just one $\lambda>0$.

Another important and useful fact about the mapping $\widetilde{J}$ is that the mapping $(\lambda T+\widetilde{J})^{-1}$ is continuous for all $\lambda>0$. Actually, it is Hölder continuous. In fact, from

$$
\begin{aligned}
\|(\lambda u+\widetilde{J} x)-(\lambda v+\widetilde{J} y)\|\|x-y\| & \geq\langle(\lambda u+\widetilde{J} x)-(\lambda v+\widetilde{J} y), x-y\rangle \\
& \geq \frac{1}{2^{p}}\|x-y\|^{p+2},
\end{aligned}
$$

for any $x, y \in D(T)$ and any $u \in T x, v \in T y$, we obtain

$$
\left\|(\lambda T+\widetilde{J})^{-1} u-(\lambda T+\widetilde{J})^{-1} v\right\| \leq 2^{p /(p+1)}\|u-v\|^{1 /(p+1)}, u, v \in H .
$$

In order to demonstrate the usefulness of the mapping $\widetilde{J}$ in the perturbation theory of monotone operators in $H$, we shall establish a surjectivity criterion, in the spirit of Theorems 2 and 6 , whose proof utilizes the function $\widetilde{J}$.

Theorem 12. Let $T: H \supset D(T) \rightarrow 2^{H}$ be maximal monotone and (a) $C: D(T) \rightarrow$ $H$ compact. Assume that there exist $\beta \in \Gamma$ and $Q>0$ such that: for every $x \in D(T)$ with $\|x\| \geq Q$ and every $v \in T x$ we have

$$
\langle v+C x, x\rangle \geq-\beta(\|x\|)\|x\|^{p+2},
$$

where $p$ is a nonnegative constant. Then $R(T+C+\epsilon \widetilde{J})=H$, for every $\epsilon \in(0,1)$. Assume, further, that

$$
\liminf _{\substack{\|x\| \rightarrow \infty \\ x \in D(T)}} \frac{|T x+C x|}{\|x\|^{p+1}}>0 .
$$


Then $\overline{R(T+C)}=H$. Let one of the following conditions hold:

(b) $C: \overline{D(T)} \rightarrow X$ is completely continuous;

(c) instead of (a), $C: \overline{D(T)} \rightarrow H$ is continuous and bounded and $(T+\widetilde{J})^{-1}$ is compact.

Then $R(T+C)=H$.

Proof. We consider the stabilizing problem

$$
T x+C x+\epsilon \widetilde{J} x \ni s,
$$

where $s$ is a fixed point in $H$, and the relevant homotopy inclusion

$$
t(T x+C x+\epsilon \widetilde{J})+(1-t) \widetilde{J} x \ni t s .
$$

We may assume that $p>0$. The case $p=0$ is covered by Theorem 6 . We only sketch the proof because of the similarity between the mappings $J$ and $\widetilde{J}$ in terms of being homeomorphisms, of type $\left(S_{+}\right)$, etc. Also, the spaces $H$ and $H^{*} \equiv H$ are uniformly convex. We note that in the two occasions where the expression $\left(\left\|x_{n}\right\|-\left\|x_{0}\right\|\right)^{2}$ guaranteed the boundedness of the sequence $\left\{x_{n}\right\}$, in the proof of Theorem 6 , we must now consider the alternate expression $\left(1 / 2^{p}\right)\left\|x_{n}-x_{0}\right\|^{p+2}$, which gives us the same result. The mapping $H(t, u)$ in Theorem 6 is now replaced by the same mapping, but with $\widetilde{J}$ in place of $J$. It is again true that $H(t, u)$ is a homotopy of compact transformations and that the degree $d(I-H(t, \cdot), B, 0)$ is well-defined and equals 1 , as in the proof of Theorem 6 , because all solutions of $u-H(t, u)=0$ are bounded by a constant which is independent of the parameter $t \in[0,1]$. To see the latter, we let $\left\{t_{m}\right\},\left\{u_{m}\right\},\left\{x_{m}\right\}$ be as in the corresponding part of the proof of Theorem 2 with $\left\|u_{m}\right\| \rightarrow \infty$ and $\left\|x_{m}\right\| \rightarrow \infty$. Using our hypotheses, there exist $\beta \in \Gamma$ and $Q>0$ such that: for every $m \geq 1$ such that $\left\|x_{m}\right\| \geq Q$, we have

$$
\begin{aligned}
\left\langle v_{m}+C x_{m}-s, x_{m}\right\rangle & \geq-\left\langle s, x_{m}\right\rangle-\beta\left(\left\|x_{m}\right\|\right)\left\|x_{m}\right\|^{p+2} \\
& \geq-\frac{\|s\|}{\left\|x_{m}\right\|^{p+1}}\left\|x_{m}\right\|^{p+2}-\beta\left(\left\|x_{m}\right\|\right)\left\|x_{m}\right\|^{p+2} \\
& =-\widetilde{\beta}\left(\left\|x_{m}\right\|\right)\left\|x_{m}\right\|^{p+2}
\end{aligned}
$$

for every sequence $\left\{v_{m}\right\}$ of terms $v_{m} \in T x_{m}$, where $\widetilde{\beta}$ is an obvious function in $\Gamma$. For such an integer $m$, we obtain

$$
\begin{aligned}
\left\langle\epsilon \widetilde{J} x_{m}, x_{m}\right\rangle=\epsilon\left\|x_{m}\right\|^{p+2} & \leq\left[1-t_{m}(1-\epsilon)\right]\left\|x_{m}\right\|^{p+2} \\
& \leq-t_{m}\left\langle v_{m}+C x_{m}-s, x_{m}\right\rangle \\
& \leq t_{m} \widetilde{\beta}\left(\left\|x_{m}\right\|\right)\left\|x_{m}\right\|^{p+2} .
\end{aligned}
$$

This inequality holds for all large integers $m$. It implies

$$
\epsilon \leq \widetilde{\beta}\left(\left\|x_{m}\right\|\right) .
$$

Since the right-hand side above converges to zero as $m \rightarrow \infty$, we have a contradiction. We omit the rest of the proof, which follows from that of Theorem 2. 
Naturally, a result like Theorem 12 most probably holds for maximal monotone operators $T: X \supset D(T) \rightarrow 2^{X^{*}}$. In its proof, we should make use of the mapping $U$ defined above. It is obvious that the mapping $\widetilde{J}$ is a homeomorphism in a general Banach space $X$ as well. It is thus natural to ask whether a theorem like Theorem 12 actually holds for $m$-accretive operators $T: X \supset D(T) \rightarrow 2^{X}$. This would certainly be true if the mapping $\widetilde{J}$ has the right coercivity properties in a general Banach space.

Open Problem 4. Let $X$ be a real Banach space and $p$ a positive constant. Prove or disprove the following statement. There is a positive constant a $(p)$ such that: for every $x, y \in X$ there exists $j \in J(x-y)$ such that

$$
\left\langle\|x\|^{p} x-\|y\|^{p} y, j\right\rangle \geq a(p) \mid\|x\|-\|y\|^{p+2} \text {. }
$$

Although the use of the mapping $\widetilde{J}$ seems rather promising in applications, it might not work with problems involving "ranges of sums." In fact, the usual applications of this theory to problems of the type int $(R(T)+R(C)) \subset \operatorname{int} R(T+C)$ make use of the uniform boundedness principle in an argument that works, to the best of our knowledge, only if $p=0$ in the definition of $\widetilde{J}$ (for some recent results, see [17] and [24]).

\section{Discussion. Possible Applications}

The transition from the solution of the problem $(T+C) x+\epsilon x \ni 0$ to a solution of the problem $(T+C) x \ni 0$ may also be achieved via a coercivity-type condition on the operator $T$. The same remark applies to the maximal monotone case.

Theorem 1 does have a maximal monotone analogue. We state it for the sake of completeness and for future reference.

Theorem 13. Let $G$ be a bounded, open and convex subset of $X$. Let $T: X \supset$ $D(T) \rightarrow 2^{X^{*}}$ be maximal monotone and (i) $C: \bar{G} \rightarrow X^{*}$ compact. Assume that $D(T) \cap G \neq \emptyset$ and that $[J-(T+C)](D(T) \cap \partial G) \subset J \bar{G}$. Then $0 \in \overline{R(T+C)}$. Assume that one of the following conditions holds:

(a) $C$ is completely continuous;

(b) instead of (i), let $J_{1}$ be compact and $C: \bar{G} \rightarrow X^{*}$ continuous and bounded. Then $0 \in R(T+C)$.

Proof. As in the proof of Theorem 6, we now consider the homotopy inclusion

$$
t(T+C+\epsilon J) x+(1-t) J x \ni 0 .
$$

The argument involving the operator $S$, in the proof of Theorem 1, is now replaced by an argument involving the operator

$$
S x \equiv J^{-1}[J x-(T+C) x]
$$

which maps $\partial G$ into $\bar{G}$. In fact, in order to obtain the contradiction involving the point $x_{t} \in \partial G$, we would now have to argue again with

$$
x_{t} \in \frac{t}{1+\epsilon t} S x_{t} .
$$


Theorem 7 is actually a special case of a more general principle which is contained in Theorem 14 below. As we mentioned in [28], in the case of $m$-accretive operators, such a result, although fruitful, might not be directly applicable in such a degree of generality. We give its statement here because it has an interesting consequence, namely Corollary 8. In Corollary 8 we impose no assumption of boundedness on the operator $C$. To avoid unnecessary complications, we assume that $0 \in D(T)$ and $0 \in T(0)$.

Theorem 14. Let $T: X \supset D(T) \rightarrow 2^{X^{*}}$ be maximal monotone with $(T+J)^{-1}$ compact, $0 \in D(T)$ and $0 \in T(0)$. Let $C: X \supset D(T) \rightarrow X^{*}$ be such that $C(\lambda T+J)^{-1}: X \rightarrow X^{*}$ is compact, for some $\lambda \in(0, \infty)$. Assume that there exists an open set $B \subset X$ such that $0 \in B, 0 \notin\left(I+\lambda J^{-1} T\right)^{-1}(\partial B)$, the set $C\left(I+\lambda J^{-1} T\right)^{-1}(\bar{B})$ is bounded and

$$
T x \not \supset 0 \text { and }\left\langle u^{*}+C x, x\right\rangle \geq 0,
$$

for all $x \in\left(I+\lambda J^{-1} T\right)^{-1}(\partial B), u^{*} \in T x$. Then $0 \in R(T+C)$.

Proof. The proof follows the steps of the proof of Theorem 7 . The main difference is that the set

$$
U=\left(I+\lambda J^{-1} T\right)^{-1}(D(T) \cap G)
$$

has now been replaced by the set $B$.

Corollary 8. Let $T: X \supset D(T) \rightarrow 2^{X^{*}}$ be maximal monotone with $(T+J)^{-1}$ compact. Let $C: X \supset D(T) \rightarrow X^{*}$ be such that $C(I+T)^{-1}: X \rightarrow X^{*}$ is compact. Assume that there exist a positive constant $b$ and $z \in D(T)$ such that $\|z\|<b, T z$ is bounded and (31) is satisfied for all $x \in D(T), u^{*} \in T x$ with $\max \left\{\|x\|,\left\|u^{*}\right\|\right\}>$ b. Then $0 \in R(T+C)$.

Proof. The proof follows exactly as in Corollary 1 of [28]. It is therefore omitted.

The compactness of the resolvents of a time-dependent $m$-accretive operator $T$ plays an important role in the compactness of the evolution operator generated by $T$. For more details on this subject, the reader is referred to the paper [26] and several of the references therein.

We are now going to exhibit various examples of partial differential as well as Nemytskii-type operators which are susceptible to the applicability of the results in this paper. We are looking for Nemytskii-type operators which are either compact or continuous and/or bounded. We are also looking for maximal monotone $(\mathrm{m}$ accretive) operators which have compact resolvents or compact inverses defined on all of $X^{*}(X)$. In these examples, $\Omega$ denotes an open, bounded and connected subset of $\mathcal{R}^{n}$ with sufficiently smooth boundary. Also, several relations hold a.e. on $\Omega$ or on $\partial \Omega$. This will be assumed without further mention. The following example was used by the author in [22].

Example 1. Consider the problem

$$
-\Delta \rho(u(x))+g(x, u(x))=p(x) \text {, a.e. } x \in \Omega .
$$

We assume the following statements.

(i) $\rho \in C(\mathcal{R}) \cap C^{1}(\mathcal{R} \backslash\{0\})$ is nondecreasing and such that $\rho(0)=0$ and, for some constants $K>0, \alpha \geq 1$,

$$
\rho^{\prime}(t) \geq K|t|^{\alpha-1}, \quad t \in \mathcal{R} \backslash\{0\} ;
$$


(ii) $g: \Omega \times \mathcal{R} \rightarrow \mathcal{R}$ is continuous and such that

$$
|g(x, u)| \leq q(x)+q_{1}|u|,
$$

where $q: \Omega \rightarrow \mathcal{R}_{+}$is in $L^{1}(\Omega)$ and $q_{1}$ is a positive constant;

(iii) $p \in L^{1}(\Omega)$ is fixed.

We let $T: L^{1}(\Omega) \supset D(T) \rightarrow L^{1}(\Omega)$ be defined by $(T u)(x) \equiv-\Delta \rho(u(x))$, where $D(T)=\left\{u \in L^{1}(\Omega) ; \rho(u) \in W_{0}^{1,1}(\Omega), \Delta \rho(u) \in L^{1}(\Omega)\right\}$. The operator $C$ is defined by $(C u)(x)=g(x, u(x))$. Bénilan showed in [3] that $T$ is $m$-accretive and $\overline{D(T)}=L^{1}(\Omega)$, while Vrabie showed in [40, Lemma 2.6.2] that $T$ generates a compact semigroup on $\overline{D(T)}$. The latter implies that $(T+I)^{-1}$ is a compact operator on $L^{1}(\Omega)$. Vainberg's Theorem 19.1 in [39] says that the operator $C$ is continuous and bounded on all of $L^{1}(\Omega)$.

From Massabo and Stuart [33] (cf. also Ding and the author [11]), we have the following example.

Example 2. Consider the problem

$$
-\Delta u(x)+q(x) u(x)+g(x, u(x), \nabla u(x))=0, \quad x \in \mathcal{R}^{n},
$$

where $n>2$, under the following assumptions.

(1) $q: \mathcal{R}^{n} \rightarrow \mathcal{R}$ is continuous and

$$
0<\inf _{x \in \mathcal{R}^{n}} q(x) \leq \sup _{x \in \mathcal{R}^{n}} q(x)<+\infty .
$$

(2) $g: \mathcal{R}^{2 n+1} \rightarrow \mathcal{R}$ is continuous and satisfies the following two conditions.

(2a) There exist constants $p \in[1, n /(n-2)), c \in \mathcal{R}_{+}$and a continuous function $g \in L^{2}\left(\mathcal{R}^{n}\right)$ such that

$$
|g(x, \eta)| \leq g(x)+c\|\eta\|^{p}, \quad(x, \eta) \in \mathcal{R}^{n} \times \mathcal{R}^{n+1},
$$

where $\|\eta\|$ denotes the Euclidean norm of $\eta$.

(2b) For every $\epsilon>0$ there exist constants $p=p(\epsilon) \in[1, n /(n-2))$ and $l=l(\epsilon) \geq 0$ such that

$$
|g(x, 0)-g(x, \eta)| \leq \epsilon\|\eta\|^{p}
$$

for every $x \in \mathcal{R}^{n}$ with $\|x\| \geq l$ and all $\eta \in \mathcal{R}^{n+1}$.

The operators $T: W^{2,2}\left(\mathcal{R}^{n}\right) \rightarrow L^{2}\left(\mathcal{R}^{n}\right)$ and $C: W^{2,2}\left(\mathcal{R}^{n}\right) \rightarrow L^{2}\left(\mathcal{R}^{n}\right)$ are defined by $(T u)(x) \equiv-\Delta u(x)+q(x) u(x)$ and $(C u)(x) \equiv g(x, u(x), \nabla u(x))$, respectively. The operator $T$ is self-adjoint, $m$-accretive, strongly accretive, and such that $T^{-1}: L^{2}\left(\mathcal{R}^{n}\right) \rightarrow W^{2,2}\left(\mathcal{R}^{n}\right)$ is a $Q^{-1}$-set-contraction. Here,

$$
Q \equiv \inf \sigma_{e}(T) \in(0, \infty)
$$

where $\sigma_{e}(T)$ is the essential spectrum of $T$. As Massabo and Stuart have shown in [33], the operator $C$ is compact.

From the Hirano and Kalinde paper [19] (cf. also the paper of the author [28]), we have Example 3 below. 
Example 3. Consider the nonlinear elliptic boundary value problem

$$
\left\{\begin{array}{l}
-\sum_{i=1}^{n} \frac{\partial}{\partial x_{i}} a_{i}\left(\frac{\partial u}{\partial x_{i}}\right)+g(x, u(x), \nabla u(x))=p(x), \quad x \in \Omega \\
u(x)=0, \quad x \in \partial \Omega
\end{array}\right.
$$

where $p \in X=L^{2}(\Omega)$. We assume that $a_{i} \in C^{1}(\mathcal{R})$ and $0<\inf _{t \in \mathcal{R}} a_{i}^{\prime}(t) \leq$ $\sup _{t \in \mathcal{R}} a_{i}^{\prime}(t)<\infty, i=1,2, \ldots, n$. We define the operator $T$ by

$$
T u=-\sum_{i=1}^{n} \frac{\partial}{\partial x_{i}} a_{i}\left(\frac{\partial u}{\partial x_{i}}\right), \quad u \in D(T) \equiv H^{2}(\Omega) \cap H_{0}^{1}(\Omega) .
$$

The operator $T$ is $m$-accretive (or maximal monotone) in $X=L^{2}(\Omega)$. Let $g \in$ $C\left(\Omega \times \mathcal{R} \times \mathcal{R}^{n}, \mathcal{R}\right)$ be such that there exist positive constants $c_{1}, c_{2}$ such that

$$
|g(x, s, t)| \leq c_{1}|s|+c_{2}, \quad(x, s, t) \in \Omega \times \mathcal{R} \times \mathcal{R}^{n} .
$$

Define the operator $C$ by

$$
(C u)(x)=g(x, u(x), \nabla u(x)), \quad(u, x) \in D(T) \times \Omega .
$$

Then $(g 1)$ implies that $C: D(T) \rightarrow X$ is bounded. We also have that the mapping $(I+T)^{-1}$ maps bounded sets of $L^{2}(\Omega)$ into bounded sets of $H^{2}(\Omega)$. Recalling that $H^{2}(\Omega)$ is compactly embedded in $H^{1}(\Omega)$, we find that $C(I+T)^{-1}$ is a compact mapping on $X$.

Example 4 can be found in Gupta and Hess [18] and Guan and the author [17].

Example 4. Let $\gamma$ be a maximal monotone graph in $\mathcal{R}^{2}$ with $0 \in \gamma(0)$ and let

$$
\mathcal{A}=-\sum_{i, j=1}^{n} \frac{\partial}{\partial x_{i}}\left(a_{i j} \frac{\partial}{\partial x_{j}}\right)
$$

be a uniformly elliptic operator with coefficients $a_{i j}$ lying in the space $C^{1}(\bar{\Omega}, \mathcal{R})$ for every $i, j=1,2, \ldots, n$. With this operator we associate the maximal monotone operator $T$ in $L^{2}(\Omega)$ defined by

$$
\begin{gathered}
D(T)=\left\{u \in H^{2}(\Omega):-\frac{\partial u}{\partial n_{\alpha}} \in \gamma(u) \text { on } \partial \Omega\right\}, \\
T u=\mathcal{A} u, \quad u \in D(T) .
\end{gathered}
$$

Here, $\frac{\partial u}{\partial n_{\alpha}}$ is the outward normal derivative given by

$$
\frac{\partial u}{\partial n_{\alpha}}=\sum_{i, j=1}^{n} a_{i j} \frac{\partial u}{\partial x_{j}} \cos \left(\bar{n}, x_{i}\right)
$$

where $\bar{n}$ is the outward normal to $\partial \Omega$. 
For the operator $C$, we assume that

$$
(C u)(x)=g_{1}(u(x))+g_{2}(x, u(x)),
$$

where $g_{1}$ is as in Gupta and Hess [18], $\beta \in \Gamma$ is continuous and $g_{2}$ satisfies the following condition:

(i) $g_{2}: \bar{\Omega} \times \mathcal{R} \rightarrow \mathcal{R}$ is continuous and there exist positive constants $c, d$ such that

$$
\left|g_{2}(x, t)\right| \leq c+d|t|, \quad(x, t) \in \Omega \times \mathcal{R}
$$

We define the operators $C_{1}(u), C_{2}(u)$ by $\left(C_{1} u\right)(x)=g_{1}(u(x))$ and $\left(C_{2} u\right)(x)=$ $g_{2}(x, u(x)), x \in \Omega, u \in L^{2}(\Omega)$, respectively.

As in Gupta and Hess [18], the operator $T_{1} \equiv T+C_{1}: L^{2}(\Omega) \supset D(T) \rightarrow 2^{L^{2}(\Omega)}$ is maximal monotone. Also, the operator $C_{2}: L^{2}(\Omega) \rightarrow L^{2}(\Omega)$ is continuous and bounded, by well-known facts on Nemytskii operators. Gupta and Hess showed in [18] that the operator $T_{1}$ is actually boundedly inversely compact, i.e., for every bounded $Q \subset X$ and every bounded $Q^{*} \subset X^{*}$ the set $Q \cap T_{1}^{-1}\left(Q^{*}\right)$ is relatively compact in $X$. This property was then used in [18] to show that the operator $\left(T_{1}+I\right)^{-1}$ maps bounded sets into relatively compact sets. However, since this operator is also continuous, we have that $\left(T_{1}+I\right)^{-1}$ is a compact resolvent.

From Fitzpatrick and Petryshyn [12], we have the following example of a compact operator on a Sobolev space.

Example 5. Let $N$ denote the set of natural numbers and, for some $m \in N$, let $S_{m}$ denote the cardinality of the set

$$
\left\{\alpha=\left(\alpha_{1}, \alpha_{2}, \ldots, \alpha_{n}\right) \in(N \cup\{0\})^{n}:|\alpha| \leq m\right\},
$$

where $|\alpha|=\alpha_{1}+\alpha_{2}+\cdots+\alpha_{n}$. For $\xi \in \mathcal{R}^{S_{m}}$, we let

$$
|\xi|=\left(\sum_{|\alpha| \leq m}\left|\xi_{\alpha}\right|^{p-1}\right)^{1 /(p-1)}
$$

where $p \in(1, \infty)$ is fixed. Let

(i) $g(x, \xi): \Omega \times \mathcal{R}^{S_{m}} \rightarrow \mathcal{R}$ satisfy the Carathéodory conditions and be such that

$$
|g(x, \xi)| \leq k_{1}|\xi|^{p-1}+\left|h_{1}(x)\right|,
$$

where $k_{1} \geq 0$ and $h_{1} \in L^{q}(\Omega)((1 / p)+(1 / q)=1)$.

Let $X$ be a closed subspace of $W^{m, p}(\Omega)$ which contains the space $W_{0}^{m, p}(\Omega)$. For every $u, v \in X$, we define the generalized Dirichlet form $b(u, v)$ as follows:

$$
b(u, v)=\langle g(\cdot, \xi(u)), v\rangle,
$$

where, for $u \in L^{p}(\Omega), v \in L^{q}(\Omega)$,

$$
\langle u, v\rangle=\int_{\Omega} u(x) v(x) d x .
$$


Here, $\xi(u): \Omega \rightarrow \mathcal{R}^{S_{m}}$ is defined by

$$
\xi(u)(x)=\left\{D^{\alpha} u(x):|\alpha| \leq m \text { and } x \in \Omega\right\},
$$

where, for $D_{i} \equiv \partial / \partial x_{i}, i=1,2, \ldots, n, D^{\alpha} \equiv D_{1}^{\alpha_{1}} D_{2}^{\alpha_{2}} \ldots D_{n}^{\alpha_{n}}$. We define the operator $C: X \rightarrow X^{*}$ by

$$
b(u, v)=\langle C u, v\rangle, \quad u, v \in X .
$$

Fitzpatrick and Petryshyn have shown in [12] that $C$ is a compact operator.

The author wishes to express his thanks to Professor Igor V. Skrypnik, Ukrainian Academy of Sciences, for his hospitality and interest during the author's stay in Kiev.

\section{REFERENCES}

1. Y. I. Alber, On the solution of nonlinear equations with monotone operators in a Banach space, Siberian Math. J. 16 (1975), 3-11. MR 51:6512

2. V. Barbu, Nonlinear Semigroups and Differential Equations in Banach Spaces, Noordhoff Int. Publ., Leyden (The Netherlands), 1975. MR 52:11666

3. P. Bénilan, Équations d'Évolution dans un Espace de Banach Quelconque et Applications, Thése, Orsay, 1972.

4. H. Brézis, M. G. Crandall and A. Pazy, Perturbations of nonlinear maximal monotone sets in Banach space, Comm. Pure Appl Math. 23 (1970), 123-144. MR 41:2454

5. F. Browder, Nonlinear Operators and Nonlinear Equations of Evolution in Banach Spaces, Proc. Symp. Pure Appl. Math., 18, Part 2, Amer. Math. Soc., Providence, 1976. MR 53:8982

6. B. D. Calvert and C. P. Gupta, Nonlinear elliptic boundary value problems in $L^{p}-$ spaces and sums of ranges of accretive operators, Nonlinear Anal. 2 (1978), 1-26. MR 80i:35083

7. I. Cioranescu, Geometry of Banach Spaces, Duality Mappings and Nonlinear Problems, Kluwer Acad. Publ., Boston, 1990. MR 91m:46021

8. K. Deimling, Zeros of accretive operators, Manuscr. Math. 13 (1974), 365-374. MR 50:3030

9. K. Deimling, Nonlinear Functional Analysis, Springer-Verlag, New York, 1985. MR 86j:47001

10. Z. Ding and A. G. Kartsatos, Nonzero solutions of nonlinear equations involving compact perturbations of accretive operators in Banach spaces, Nonlinear Anal. 25 (1995), 1333-1342.

11. Z. Ding and A. G. Kartsatos, P-Regular mappings and alternative results for perturbations of $m$-accretive operators in Banach spaces, Topol. Meth. Nonl. Anal. (to appear).

12. P. M. Fitzpatrick and W. V. Petryshyn, On the nonlinear eigenvalue problem $T(u)=\lambda C(u)$ involving noncompact abstract and differential operators, Boll. Un. Mat. Ital. B (5) 15 (1978), 80-107. MR 80b:47083

13. Z. Guan, Ranges of operators of monotone type in Banach spaces, J. Math. Anal. Appl. 174 (1993), 256-264. MR 95b:47068

14. Z. Guan, Solvability of semilinear equations with compact perturbations of operators of monotone type, Proc. Amer. Math. Soc. 121 (1994), 93-102. MR 94g:47080

15. Z. Guan and A. G. Kartsatos, Solvability of nonlinear equations with coercivity generated by compact perturbations of $m$-accretive operators in Banach spaces, Houston J. Math. 21 (1995), 149-188. CMP 95:12

16. Z. Guan and A. G. Kartsatos, On the eigenvalue problem for perturbations of nonlinear accretive and monotone operators in Banach spaces, Nonlinear Anal. (to appear).

17. Z. Guan and A. G. Kartsatos, Ranges of perturbed maximal monotone and $m$-accretive operators in Banach spaces, Trans. Amer. Math. Soc. 347 (1995), 2403-2435. MR 95i:47096

18. C. P. Gupta and P. Hess, Existence theorems for nonlinear noncoercive operator equations and nonlinear elliptic boundary value problems, J. Diff. Eq. 22 (1976), 305-313. MR 57:13600

19. N. Hirano and A. K. Kalinde, On perturbations of $m$-accretive operators in Banach spaces, Proc. Amer. Math. Soc. (to appear).

20. D. R. Kaplan and A. G. Kartsatos, Ranges of sums and control of nonlinear evolutions with preassigned responses, J. Optim. Theory Appl. 81 (1994), 121-141. CMP 94:12 
21. A. G. Kartsatos, Zeros of demicontinuous accretive operators in reflexive Banach spaces, Integral Equations 8 (1985), 175-184. MR 86j: 47078

22. A. G. Kartsatos, On compact perturbations and compact resolvents of nonlinear m-accretive operators in Banach spaces, Proc. Amer. Math. Soc. 119 (1993), 1189-1199. MR 94c:47076

23. A. G. Kartsatos, Recent results involving compact perturbations and compact resolvents of accretive operators in Banach spaces, Proceedings of the First World Congress of Nonlinear Analysts, Tampa, Florida, 1992, Walter De Gruyter, New York, (1995), pp. 2197-2222.

24. A. G. Kartsatos, Sets in the ranges of sums for perturbations of nonlinear $m$-accretive operators in Banach spaces, Proc. Amer. Math. Soc. 123 (1995), 145-156. MR 95c: 47072

25. A. G. Kartsatos, On the construction of methods of lines for functional evolutions in general Banach spaces, Nonlinear Anal. 25 (1995), 1321-1331.

26. A. G. Kartsatos, A compact evolution operator generated by a time-dependent $m$-accretive operator in a general Banach space, Math. Ann. 302 (1995), 473-487. CMP 95:15

27. A. G. Kartsatos, Sets in the ranges of nonlinear accretive operators in Banach spaces, Studia Math. 114 (1995), 261-273. CMP 95:14

28. A. G. Kartsatos, On the perturbation theory of $m$-accretive operators in Banach spaces, Proc. Amer. Math. Soc. (to appear).

29. A. G. Kartsatos and X. Liu, Nonlinear equations involving compact perturbations of maccretive operators in Banach spaces, Nonlinear Anal. 24 (1995), 469-492. CMP 95:7

30. A. G. Kartsatos and R. D. Mabry, Controlling the space with pre-assigned responses, J. Optim. Theory Appl. 54 (1987), 517-540. MR 88j:49024

31. V. Lakshmikantham and S. Leela, Nonlinear Differential Equations in Abstract Spaces, Pergamon Press, Oxford, 1981. MR 82i:34072

32. N. G. Lloyd, Degree Theory, Cambridge Univ. Press, Cambridge, 1978. MR 58:12558

33. I. Massabo and C. A. Stuart, Positive eigenvectors of $k$-set contractions, Nonlinear Anal. 3 (1979), 35-44. MR 80b: 47073

34. M. Nagumo, Degree of mapping in convex linear topological spaces, Amer. J. Math. 73 (1951), 497-511. MR 13:150b

35. D. Pascali and S. Sburlan, Nonlinear Mappings of Monotone Type, Sijthoff and Noordhoff Intern. Publ., Alphen aan den Rijn, 1978. MR 80g:47056

36. S. Reich, Extension problems for accretive sets in Banach spaces, J. Funct. Anal. 26 (1977), 378-395. MR 57:17393

37. E. H. Rothe, Introduction to Various Aspects of Degree Theory in Banach Spaces, Math. Surveys and Monographs, 23, A.M.S., Providence, 1986. MR 87m:47145

38. I. V. Skrypnik, Methods for Analysis of Nonlinear Elliptic Boundary Value Problems, Transl. Math. Monographs, 139, A.M.S., Providence, 1994. MR 95i:35109

39. M. M. Vainberg, Variational Methods for the Study of Nonlinear Operators, Holden-Day, Inc., San Francisco, 1964. MR 31:638

40. I. I. Vrabie, Compactness Methods in Nonlinear Evolutions, Longman Sci. Tech., London, 1987. MR 90f: 47101

41. E. Zeidler, Nonlinear Functional Analysis and its Applications, II/B, Springer-Verlag, New York, 1990. MR 91b:47002

Department of Mathematics, University of South Florida, Tampa, Florida 33620-5700

E-mail address: hermes@gauss.math.usf.edu 